\title{
Improving Automatic Tuning of Hadoop and Spark by Analysing Container Performance Metrics
}

\author{
by
}

Siyu Zhou

A thesis submitted to the Faculty of Graduate and Postdoctoral Affairs in partial fulfillment of the requirements

for the degree of

\section{Master of Computer Science}

in

M.C.S Computer Science With Specialization in Data Science

\author{
Carleton University \\ Ottawa, Ontario
}

(C) 2019

Siyu Zhou 
The undersigned hereby recommends to the

Faculty of Graduate and Postdoctoral Affairs acceptance of the thesis

\title{
Improving Automatic Tuning of Hadoop and Spark by Analysing Container Performance Metrics
}

\author{
submitted by Siyu Zhou \\ in partial fulfillment of the requirements for the degree of \\ Master of Computer Science
}

Professor Amiya Nayak, Ph.D, External Examiner

Professor Jean-Pierre Corriveau, Ph.D, Examiner

Professor Frank Dehne, Ph.D, Thesis Supervisor

Professor Anil Somayaji, Ph.D, Chair

School of Computer Science

Ottawa-Carleton Institute of Computer Science

Department of School of Computer Science

Carleton University

February, 2019 


\section{Abstract}

This thesis attempts to provide further research on improving automatic tuning of Hadoop and Spark by analysing container performance metrics. Analytics frameworks, for example, Hadoop and Spark, are powerful tools in the study of big data. The parameter values in these analytics frameworks significantly affect the performance of applications. However, it is difficult to select the optimal framework parameter values for every application. Many research teams have proposed methods for the automatic tuning of Hadoop or Spark parameters from different perspectives. Most of the currently existing automatic tuning systems do not support working with different kinds of frameworks and Resource Managers. In addition, research on selecting the best timing to evoke the tuning method is lacking, which is another gap in the study of big data frameworks. Our research aims to fill these gaps.

This research introduces novel container performance metrics and proves that these metrics are beneficial in the development of automatic tuning systems. Hadoop and Spark show different patterns in the static and dynamic values of container creation rate, container completion rate, container average response time and relative standard deviation of response-time(RSD). By applying five kinds of machine learning algorithms, container creation rate was found to be the most sensitive metric to identify and classify the workload type at an average accuracy of $83 \%$. RSD can be used to detect workload transitions with an average accuracy of $74 \%$. Our research results will decrease tuning overhead and promote the development of automatic tuning systems. 
This thesis is dedicated to my parents, Buhong Zhou and Li Zhang. Thanks for your unconditional love which supports me to face all kinds of difficulties in my life. 


\section{Acknowledgments}

First and foremost I would like to express my appreciation and my sincere gratitude to my supervisor Professor Dr.Frank Dehne. Thanks for all of your guidance during my master studies. It is my honour and fortunate to be one of your students.

I would like to express my special appreciation to the Ph.D Candidate Mikhail Genkin. This research would not have been possible without his cooperation and advice. I would like to thank all the past graduate students in our lab. All the contributions you made to our research is valuable and unique.

I would like to thank Professor Dr.Jean-Pierre Corriveau, Professor Dr.Amiya Nayak, Professor Dr.Anil Somayaji for serving as my committee members.

I also want to express my appreciation to all of School of Computer Science and Institute of Data Science faculty and staff. Without such a great program, I cannot finish my master degree with joy.

Finally, I would like to thank my parents for the love they give me. Also thanks them for respecting every decision I made. 


\section{Table of Contents}

Abstract $\quad$ iii

Acknowledgments $\quad$ v

Table of Contents vi vi v

List of Tables $\quad$ ix

List of Figures $\quad$ x

1 Introduction $\quad 1$

1.1 Background and Trend of Data Science . . . . . . . . . . . . . 1

1.2 Motivation and Problem Statement . . . . . . . . . . . . . 1

1.3 Research Team Members . . . . . . . . . . . . . . . . 2

1.4 Thesis Contributions . . . . . . . . . . . . . . . 3

1.5 Thesis Organization . . . . . . . . . . . . . . . 4

2 Background 5

2.1 Induction to Hadoop, Spark, and the Resource Manager . . . . . . . 5

2.1.1 Introduction to Hadoop and YARN . . . . . . . . . . . 5

2.1.2 Introduction to Spark . . . . . . . . . . . . . . 7

2.1.3 Introduction to the Resource Manager . . . . . . . . . . . . . 9

2.2 Parameters Tuning . . . . . . . . . . . . . . . . . 9

2.2.1 Manual Tuning . . . . . . . . . . . . . . . . 10

2.2.2 Automatic Tuning ................ . 10

3 Literature Review $\quad 13$

3.1 Hadoop and Spark Automatic Tuning . . . . . . . . . . . . . . 13 
3.2 Workload Characteristic . . . . . . . . . . . . . . 15

3.3 Limitations of Previous Research . . . . . . . . . . . . . . 16

4 Overview of Kermit $\quad 17$

4.1 Design of Kermit . . . . . . . . . . . . . . . . . . . . . . 17

4.1.1 How Kermit Improves the Performance of Frameworks . . . . 17

4.1.2 Integration Kermit with YARN . . . . . . . . . . . . 19

4.2 Inspiration From Kermit . . . . . . . . . . . . . . . . 20

5 Experimental Design and Implementation 21

5.1 Design of Experiments . . . . . . . . . . . . . . 21

5.1.1 Related Concepts . . . . . . . . . . . . . . . 21

5.2 The Implementation of Experiments . . . . . . . . . . . . . . . . . . . 24

5.2 .1 Cluster Setup . . . . . . . . . . . . . . . 24

5.2.2 Parameters Setting . . . . . . . . . . . . . 25

5.2 .3 Benchmarks .................... 26

5.2.4 Experimental Procedures . . . . . . . . . . . . . . . . . 30

5.2.5 Experimental Data . . . . . . . . . . . . . . . 31

6 Data Analysis and Validation 33

6.1 Data Visualization . . . . . . . . . . . . . . . . . . 33

6.1.1 Hadoop Single Job . . . . . . . . . . . . . . . . . 33

6.1.2 Single-user Spark Job Flow . . . . . . . . . . . . . . . . 35

6.1.3 Hadoop Job Flow and Spark Multi-user Job Flow . . . . . . . 37

6.2 Statistical Analysis of Data . . . . . . . . . . . . . . . 39

6.2.1 The Analysis of Steady State Values and Dynamic Values . . . 39

6.2.2 The Analysis of Correlation Coefficient . . . . . . . . . . . . . 41

6.3 Data Validation . . . . . . . . . . . . . . . . . 43

6.3.1 Identification and Classification of the Workload Type . . . . 43

6.3.2 Detection of the Workload Transition . . . . . . . . . . . . 46

6.4 Ranking of the Metrics . . . . . . . . . . . . . . . . . . . 48

7 Implications for Online Auto-Tuning Systems 49

8 Conclusions and Future Works $\quad 51$

8.1 Conclusions .......................... 51 
8.2 Future Work . . . . . . . . . . . . . . . . . . 52

List of References $\quad 53$ 


\section{List of Tables}

5.1 Configuration of host VM. . . . . . . . . . . . . 25

5.2 Configuration of cluster nodes. . . . . . . . . . . . . 25

5.3 Assign values to parameters. . . . . . . . . . . . . . 26

5.4 Benchmark in the experiments . . . . . . . . . . . 27 


\section{List of Figures}

2.1 Architecture of Hadoop version2 and YARN . . . . . . . . . . . 6

2.2 Architecture of Spark . . . . . . . . . . . . . . . 8

2.3 Flowchart of online tuning . . . . . . . . . . . . . . . . . . . . 12

4.1 KERMIT architecture. From [1] . . . . . . . . . . . . . . . 18

4.2 How KERMIT integrates with YARN. From [1] . . . . . . . . . . 19

5.1 Windows . . . . . . . . . . . . . . . . . 22

5.2 Cluster architecture. From $[2] \ldots \ldots . \ldots . \ldots . . \ldots 24$

5.3 Cluster architecture. From $[3] \ldots \ldots$. . . . . . . . . . 27

5.4 The component of each row in TeraGen. . . . . . . . . . . . . . 28

5.5 MapReduce process in TeraSort. . . . . . . . . . . . . . . . . 29

5.6 Twenty six experiments.From [2] . . . . . . . . . . . . . . . . 31

6.1 Container performance metrics for Hadoop WordCount-TeraSort-KMeans job flows. From $[2]$. . . . . . . . . . . . . . . . . . . 34

6.2 Execution order of SMB-userCase2 . . . . . . . . . . . . 35

6.3 Container performance metrics for a sequence of machine-learning and Spark SQL jobs . . . . . . . . . . . . . . . . . . . 36

6.4 Execution order of SMB-userCase1 . . . . . . . . . . . . . . 37

6.5 Container performance metrics for multi-user Spark workload with batch and interactive query components. From [2] . . . . . . . . . . 38

6.6 Radar chart showing average Hadoop and Spark workload metric values. From [2] . . . . . . . . . . . . . . . . . . . . . . . 40

6.7 Radar chart showing average Hadoop and Spark workload transition values. From [2] . . . . . . . . . . . . . . . . . . . . . . . . 41

6.8 Flow chart of problem transformation . . . . . . . . . . . . . . . . 42

6.9 Results of correlation coefficient . . . . . . . . . . . . . . . 43

6.10 Evaluation of classifier . . . . . . . . . . . . . . . . . . . 44

6.11 Workload classification accuracy for machine-learning algorithms . . . 45 


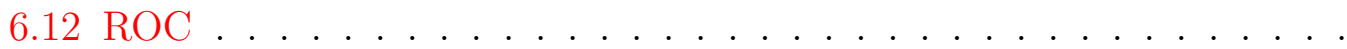

6.13 Impact of using different container performance statistics on the classification accuracy of the Random Forrest algorithm. From [2] . . . . 46

6.14 Explaination of window . . . . . . . . . . . . . . . 47

6.15 Workload transition detection accuracy for different container performance metrics. From [2] . . . . . . . . . . . . . . . . . . 48

7.1 A possible automatic tuning system architecture. From [2] . . . . . . 50 


\section{Section 1}

\section{Introduction}

\subsection{Background and Trend of Data Science}

Data science is an interdisciplinary research area at the intersection of math, computer science, and domain knowledge. It appeared in the early period of the 21st Century [4]. With the development of big data, data science has become a prevalent topic in academia and industry.

The data amount is increasing from GigaByte(GB) to ExaByte(EB) or ZettaByte(ZB) in the future. With this huge amount of data, it is crucial to improve the computing speed in order to analyse this data as quickly as possible. For example, with the aim of being able to react to fast changes in the market, business data can be used to provide real-time analysis results. However, high-performance computing with an enormous amount of data is difficult for a single computer. Big data analytic frameworks that use a computer cluster are a promising solution to this problem. Two frameworks-Hadoop and Spark are frequently employed by companies in the industry, for example, Facebook, Google, and Shopify. How to make these frameworks achieve the best performance has been the subject of a lot of research.

\subsection{Motivation and Problem Statement}

While Hadoop and Spark have hundreds of parameters, only a few dozen of them play a significant role in determining framework performances [5] [6]. Since each

parameter has a number of possible values, there are numerous combinations of these parameter values. Meanwhile, different workloads require different parameter 
values to achieve optimal performance. However, manual tuning of parameters is extremely expensive and time-consuming. In order to provide an efficient solution for parameter value selection, various automatic tuning systems (e.g JellyFish, Kermit, Mronline) were proposed by many research teams [7] [1] [8].However, most of the currently existing automatic tuning systems do not support working with different kinds of frameworks and the Resource Managers.

For an automatic tuning system, it is essential to avoid tuning overhead, especially when frameworks work with multi-user job flows. This requires the tuning systems to be able to choose the proper time to evoke the tuning method. Generally, when workload transitions happen, the optimal parameter values for the current workload will be different from the previous one, which means the tuning method should be evoked at this moment.

Motivated by the above reasons, we researched whether container performance metrics - measures of performance for memory, central processing unit(CPU), disk space, etc in the constructor - could be used to identify and classify the workload type, as well as detect workload changes which lead to improving the efficiency of automatic tuning systems over the basic tuning. By detecting workload changes, auto-tuning systems can evoke the tuning method at appropriate time and avoid tuning overhead. According to the current workload type, a specific tuning strategy will be selected to do automatic tuning. Based on our analytical results, we also researched whether a prototype of automatic tuning system could be proposed.

\subsection{Research Team Members}

The research work presented in this thesis is the result of years of research and valuable contributions from each team member. The team members are listed below:

- Mikhail Genkin

- Frank Dehne

- Pablo Navarro

- Siyu Zhou 
The author of this thesis believes everyone in the team has made unique and valuable contributions to the research results.

\subsection{Thesis Contributions}

The results of this research had been presented on Special International Symposium on Benchmarking, Measuring and Optimizing in 2018. The contributions are listed ad follows:

- The following four kinds of container performance metrics were studied in this thesis

1. Container creation rate

2. Container completion rate

3. Container average response time

4. Relative standard deviation of response-time(RSD)

- Experiments were run on Hadoop and Spark which means the methodology covered in this thesis is compatible and possible be integrated with other frameworks.

- The experiments are designed to include multi-user jobs which are closer to real-life analytics scenarios.

- The data scale used in this research is in TeraBite(TB) while most other research teams use data on the scale of GB. Our data scale is a better approximation of the situation in industry.

- Container creation rate is the most sensitive metric in identifying and classifying the workload type. After applying fives kinds of machine learning algorithms to do classification, the highest average accuracy was $83 \%$.

- In detecting workload transitions, RSD was proved to be the most sensitive metric with a highest accuracy of $74 \%$.

- This research ranked the importance of the four container performance metrics according to the results of data validation. 
- The results of this research could be used in the development of on-line automatic tuning systems

\subsection{Thesis Organization}

1. Section 2 introduces the architecture of Hadoop, Spark, and the Resource Manager. This section also gives an overview of manual tuning, offline auto-tuning and online auto-tuning. Based on this section, the readers will understand how parameter tuning works in the analytics frameworks.

2. Section 3 reviews the state of the art of tuning analytics frameworks and features of workloads.

3. Section 4 presents an overview of Kermit and the inspiration gained from Kermit.

4. Section 5 explains how the experiments were performed. The related concepts and benchmarks are also introduced in this section.

5. Section 6 analyses the experimental data from quantitative and qualitative views. Machine learning algorithms and Welch's t-test are applied to select the most sensitive metric in classifying the workload type and detecting workloads transitions, respectively.

6. Section 7 explains a possible online automatic tuning system inspired by the conclusions from the results of our research.

7. Section 8 concludes this thesis and discusses future work. 


\section{Section 2}

\section{Background}

\subsection{Induction to Hadoop, Spark, and the Resource Manager}

Nowadays, Hadoop and Spark are popular big data analytics frameworks. They are designed on distributed data storage and parallel computing, which allows them to work with huge amounts of data efficiently. The Resource manager(RM), which is responsible for fully utilising and assigning resources in the cluster, is the component that can be integrated with Hadoop and Spark. In this subsection, the architecture of Hadoop and Spark will be introduced.

\subsubsection{Introduction to Hadoop and YARN}

Hadoop is a master and worker nodes computing framework. The distributed data storage system in Hadoop is Hadoop Distributed File System(HDFS) which is developed based on the classic research paper, Google File System [9]. The parallel computing system in Hadoop is called MapReduce which is based on another research paper, "MapReduce" [10]. MapReduce can move the computation to data which is the main reason that leads to improving the computing efficiency of Hadoop than the method of moving data to the computation.

At the start of this research, Hadoop had two versions. In Hadoop Version1, JobTracker is responsible for assigning resources, scheduling the job and monitoring the job. Since JobTacker is in single mode and is multi-purpose, it decreases the scalability of Hadoop. Hadoop Version2 solved this problem by decoupling JobTraker 
into the Resource Manager and Application Manager by using Yet Another Resource Negotiator(YARN). Figure 2.1 shows the architecture of Hadoop Version2 and YARN.

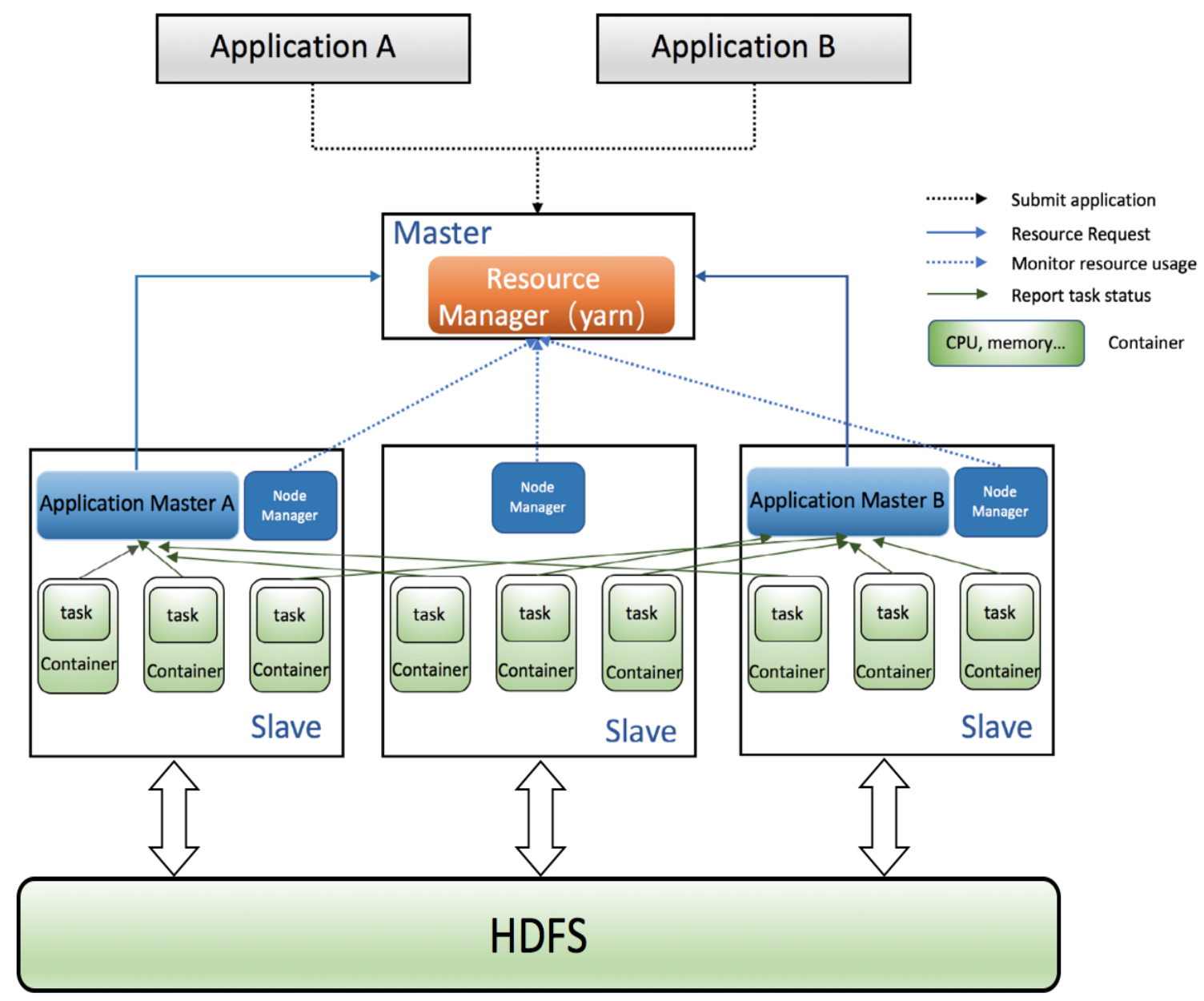

Figure 2.1: Architecture of Hadoop version2 and YARN

From Figure 2.1, the process of Hadoop application execution [11] is summarised as follows:

1. Client submits the application to the master node.

2. RM launches application master (AM) in the worker nodes.

3. AM sends resource requests to RM.

4. AM and Node Manager(NM) launch containers in every slave node. NM monitors the resource utilization in containers. 
5. Each container executes one task based on the User-defined code.

6. AM reports the application execution status to Client.

7. All the tasks are finished. AM deregisters from RM, and RM is shut down.

\subsubsection{Introduction to Spark}

Spark is an open-source cluster-based computing framework [12]. It was developed by UC Berkeley Academic Personnel Manual (APM) lab and was donated to Apache Software Foundation later [12]. After research on the official Spark document [13] and other resources [14] [15] [16], the overview of the Spark Architecture in cluster mode is introduced in this subsection.

Spark also works in master and worker nodes mode. The Spark application can contain one single job or multiple interactive jobs. When the client submits an application, user-defined code maps into the Spark drive and executor. Figure 2.2 shows the architecture and workflow of Spark.

After the client submits a Spark application, SparkContext will be started by the main() function. Each application has one SparkContext which is active for the whole duration of the application execution and allocates resources within this application. Cluster Manager assigns executors to every worker node. Every executor starts to work based on the user-defined application code sent by SparkContext.

In SparkContext, based on resilient distributed dataset(RDD) and Directed Acyclic Graph Scheduler(DAG), jobs are divided into stages. The stage consists of a set of tasks which share the same user-defined code in the running time. After stages are submitted to TaskScheduler, each task will be distributed among the executors. Executors are responsible for executing each task and finishing jobs. To track the status of executors, SparkContext communicates with executors through a periodical heartbeat. 


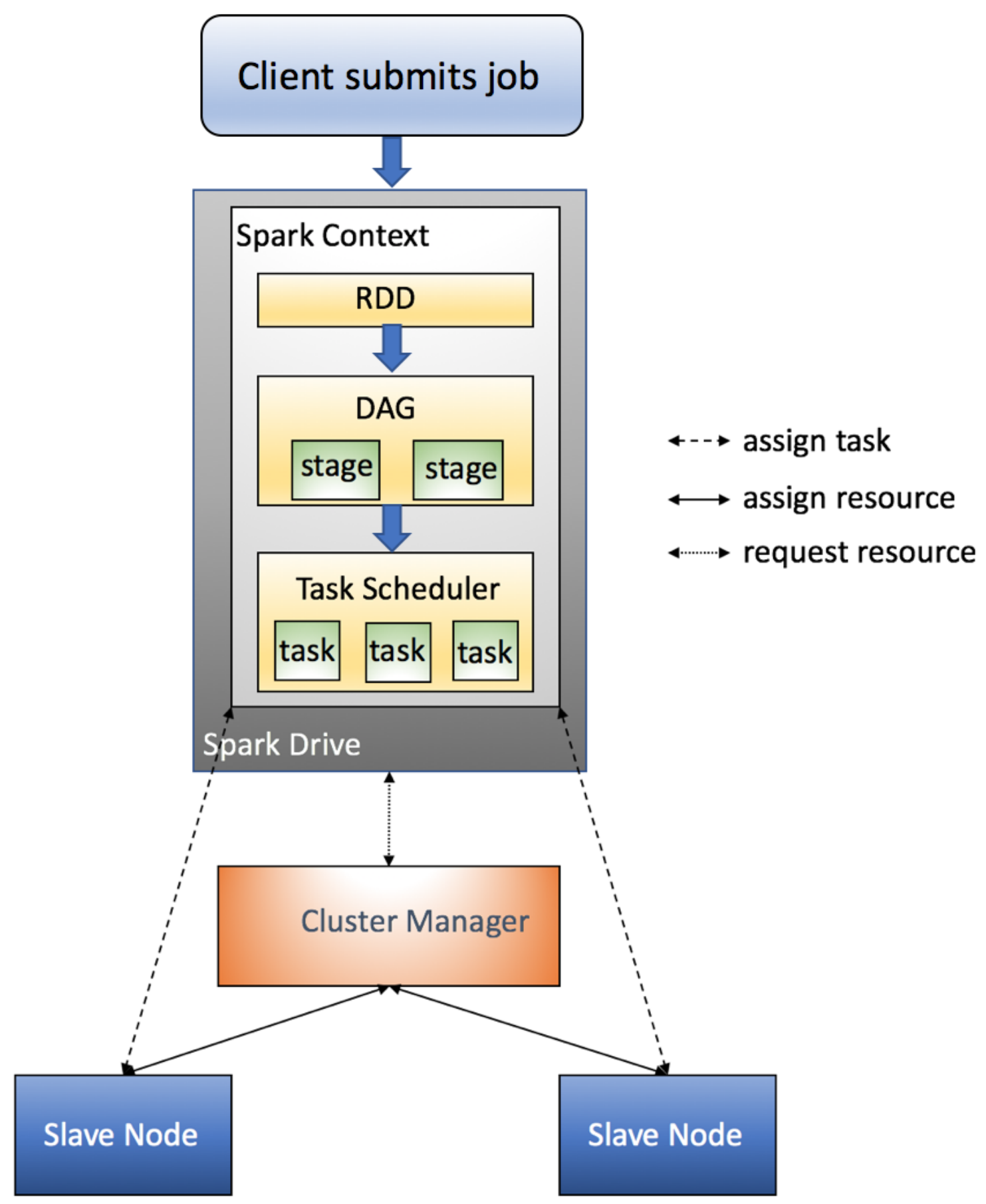

Figure 2.2: Architecture of Spark

In Hadoop, since all the data is stored on the disk, Hadoop spends over $90 \%$ of the process time doing reads and writes in HDFS. Since RDD can provide in-memory data computing, this allows Spark to run up to 100 times faster than Hadoop. The 
lazy evaluation in DAG can optimise the execution plan, speeding up the computing even though the data persists on the disk.

\subsubsection{Introduction to the Resource Manager}

Hadoop and Spark can use different kinds of open source the Resource Managers to improve the efficiency of resources utilisation.

The resource manager assigns resources to containers, then the tasks will be run inside of the containers. The container in this research refers to a construct of resources rather than a Docker container. The details of a container are explained in Section 5.1.1.1. Figure 2.1 and the last paragraph of Section 2.1.1 show how yarn works in Hadoop. Spark leverages YARN in a similar way but with some differences. In Spark, executors run as containers and tasks run inside of executors. While Hadoop releases containers when a single task is finished, Spark releases the executors after the whole job is completed.

Now, there are many kinds of the Resource Managers, for example, YARN, Mesos, and Kubernetes. Since YARN can work with Hadoop and Spark stably, it was selected for our research to prove that our results are generic. Regardless of what kind of RM is integrated with the analytics framework, the containers will be responsible for executing tasks. Since this research work focuses on container performance metrics, the results will be adaptable to not only YARN but also other RMs.

\subsection{Parameters Tuning}

According to past research [5] [6], the values of parameters determine the performance of analytics frameworks. For Hadoop, it has hundreds of parameters, but only dozens of them significantly affect the performance of Hadoop. Other frameworks are also faced with a similar situation. In order to improve the efficiency of analytics frameworks, the optimal values of parameters should be assigned before running the applications. This leads to a research question of how to select the optimal values of parameters. This subsection will introduce different kinds of parameters used in the 
tuning methods for Hadoop and Spark. After the elaboration provided in this subsection, the importance and reasoning of automatic tuning will be clearly understood by the readers.

\subsubsection{Manual Tuning}

In manual tuning, there are experts responsible for selecting the optimal parameter values of the framework in order to make current application achieve the best performance with the current framework. The experts start with some parameter values as initial guess and collect performance data. After experts analyzed the data, they decide which parameters should be modified and how much the values need to be changed. Then the experts run the application with the new parameter values again and collect performance data for analysis. This process needs to be repeated several times until satisfied performance is achieved. Some of the drawbacks of manual tuning are the following:

1. Sub-optimal Values - Since each parameter has many possible values, there will be numerous combinations of these parameter values. It is impossible for the experts to try every combination and select the optimal combination. Often, the selection will stop once a satisfactory performance is reached.

2. Time-Consuming - If the data size of the application is changed, the optimal values of the parameters will also change. This means that the experts need to do the whole process of manual tuning again even if the application type does not change. In manual tuning, in order to collect frameworks performance data for different combinations of parameter values, the experts need to run the application for each combinations. The number of combinations is enormous which makes it time-consuming to search for the optimal values.

\subsubsection{Automatic Tuning}

In order to solve the restrictions of manual tuning and improve the performance of analytics frameworks, automatic tuning has been explored by many research teams. An auto-tuning system leverages algorithms to analyse performance data and select optimal values as opposed to the experts doing it by hand. The automatic tuning includes two categories: off-line auto-tuning and on-line auto-tuning. 


\subsubsection{Offline Auto-tuning}

The offline auto-tuning system needs to restart the cluster to modify the values of parameters. Based on the research paper, "Mronline" [8], the process of offline autotuning is summarised as follows:

1. Several tests are running with initial parameter values and modified values.

2. Job performance and system resource usage logs are collected.

3. The data obtained in step 2 is used to analyse and generate new values for parameters.

4. The framework is shut down and new values are set for the parameters in the configuration files.

5. The framework is started and the application is run.

6. Steps 1 to 5 are repeated until a satisfied performance is achieved.

In comparison to manual tuning, offline auto-tuning leverages algorithms that have a higher possibility of finding optimal parameter values. However, drawback 2 discussed in Section 2.2.1 is not solved by offline tuning. Offline-tuning methods only test one combination of configuration values in each round of job execution. The job needs to be executed many times to collect performance data. This situation gets worse when the job needs a long time to finish one round execution.

"Mronline", "HAT", "Gunther" are different offline auto-tuning methods which are task history-based, search-based, cost-based, respectively [17] [18] [19] .

\subsubsection{Online Auto-tuning}

An online auto-tuning system does not need to restart the framework when modifying the parameter values, which is the most significant difference from an offline auto-tuning system. Basically, the online auto-tuning system leverages a built-in component to analyse performance data, search new values of parameters and assign these parameter values to the frameworks. Figure 2.3 shows a generalized overview of how to do online auto-tuning. Different research teams have proposed different methods of implementation. 


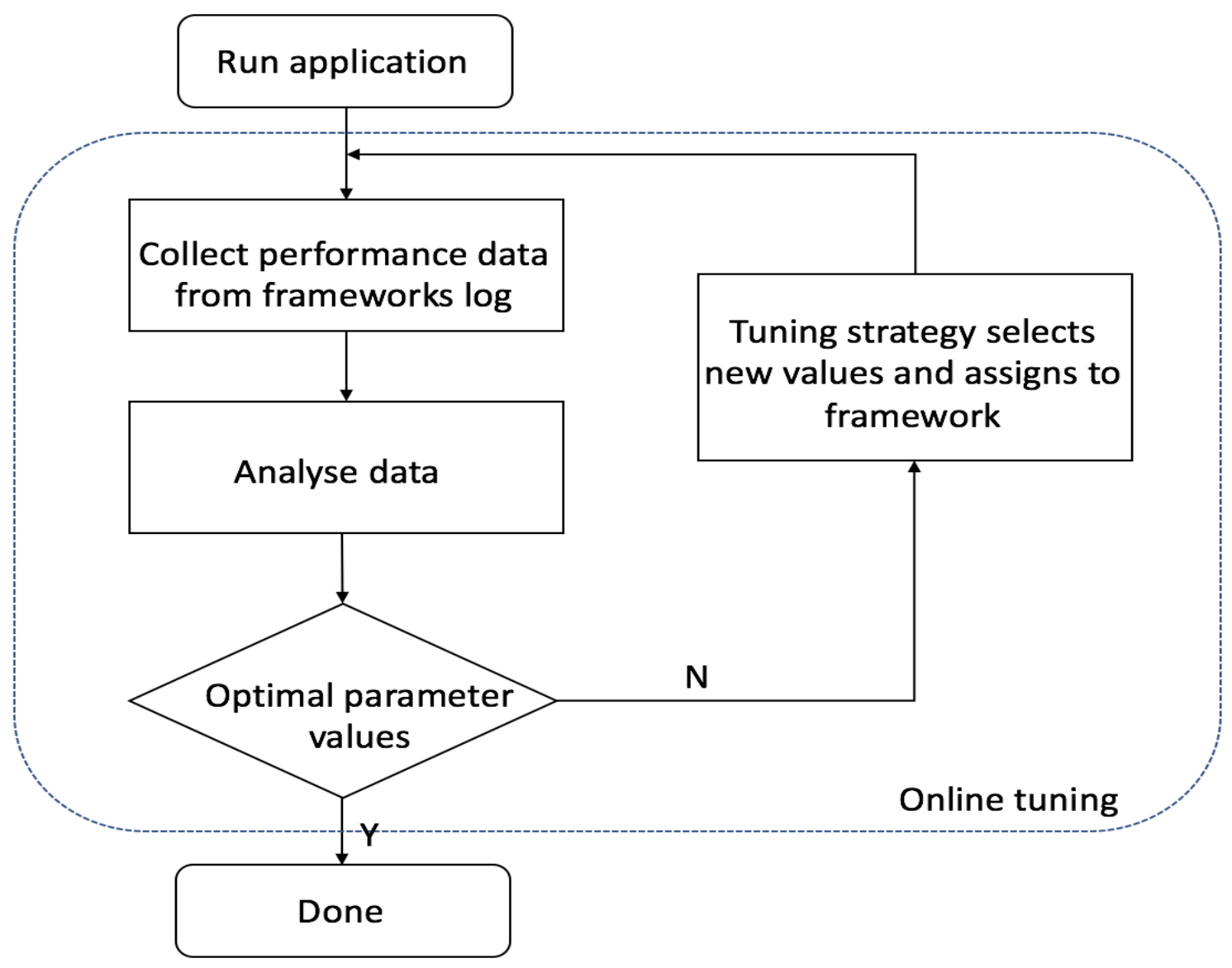

Figure 2.3: Flowchart of online tuning

"Mronline", "Kermit" proposed different online-tuning methods [8] [1]. In these two methods, they can try many combinations of parameter values in one run of application execution which solves drawback 2 of offline auto-tuning discussed in Section 2.2.1. 


\section{Section 3}

\section{Literature Review}

In this research, by analysing container performance metrics, the workload type can be identified, and workload transitions can be detected. The results of this research will lead to improving the efficiency of automatic tuning in analytics frameworks such as Hadoop and Spark over the basic tuning. The literature review will discuss automatic tuning and workload characterisation.

\subsection{Hadoop and Spark Automatic Tuning}

Automatic tuning is a hot topic in the data analytics framework research area. Different research teams have proposed different methods for tuning. Some research teams [20] [21] [22] [17] [18] [19] [23] [24] [7] [25] [26] do the tuning for Hadoop only while other teams [27] [28] [29] focus on Spark only. Very few teams tune for both Hadoop and Spark [1] [30]. Four papers about automatic tuning were selected and are introduced in this subsection. We selected these four is because they research on Hadoop only, Spark only, Spark only and both Spark and Hadoop respectively. These four paper provides the comprehensive explanation of automatic tuning for different frameworks.

JellyFish [7] is an online tuning method for Hadoop. JellyFish leveraged elastic container which is different from the type of container used in our research. According to the statistics of the current task, JellyFish utilised a divide-and-conquer algorithm and a model-based hill climbing algorithm to search and selected the best configurations for the current task. In order to reduce search space, it classified parameters into three categories: map phase parameter, reduce phase parameter 
and both map and reduce phase parameter. In the experiments, seven workloads were running with data size up to $100 \mathrm{~GB}$. As a result, JellyFish achieved $24 \%$ and $65 \%$ average performance improvement over default settings for the first-time job execution and subsequent executions, respectively.

Based on the binary classification, and multi-classification, Wang et al. [27] tuned Spark parameters. In this research, binary classification is used to compare whether input data or default parameter values reduce the Spark application execution time. If the execution time is shorter for the input data, then multi-classification will be invoked. According to the percentage decrease of the execution time, input data will be classified into five categories based on a multi-classification machine learning model. In these models, thirteen Spark parameters, such as number of cores and memory used, are tuned for four workloads which are Sort, WordCount, Grep, NaivesBayes. They claim that the Decision Tree algorithm has the best performance when they use the following evaluation metrics: the duration for building model, the duration for prediction and prediction accuracy. This research used the Recursive Random Search algorithm to search for the optimal combination of parameter values. The maximum data size used in their experiment is $10 \mathrm{~GB}$. Their results showed that this method achieves a $36 \%$ average decrease in execution time when compared with default settings.

Jia and colleagues [28] focused on auto-tuning the number of parallel threads in Spark workloads using a Power 8 processor based on machine learning algorithms in 2016. They claim that this method can be applied to Hadoop and Flink. In the offline training phase, twenty Spark workloads with different simultaneous multithreading(SMT) configurations were executed to collect micro-architecture level data. This data and machine learning algorithms were used to construct prediction models. In the online prediction phrase, by invoking a prediction hook at every stage, nine Spark application configurations were tuned. In every stage, according to the current SMT configuration in the current task, the corresponding model was selected to predict the optimal number of threads for the upcoming task. Compared with the default configuration of eight threads, their method resulted in an improvement of $16.2 \%$ in average performance. 
MR-Advisor [25] is a tuning tool which can apply to both Hadoop and Spark, based on the user's input information. The input information includes the cluster information of Hadoop and Spark, a range of parameter values, and benchmarks. MR-Advisor maps the input parameters of Map-Reduce into appropriate framework configuration parameters. This parameter space allows MR-Advisor to be used to tune both Hadoop and Spark. MR-Advisor can also work with different file systems such as HDFS and Tachyon. When all submitted jobs finish, MR-Advisor collects the execution times and uses a divide-and-conquer algorithm to select the optimal result and the corresponding best configuration. In the experiments of this research work, the maximum data size used was a 100GB.

\subsection{Workload Characteristic}

Only a few teams work on the analysis of features for both Hadoop and Spark workloads. References [31] [3] [32] [33] [34] [35]were reviewed by the author of this thesis. Two selected papers [33] [34] are reviewed in this subsection. These two paper have high citation number.

Awan et al. [33] compared and characterised batch and stream workloads on Spark. Workloads from Spark Core, Spark MLib, Graph X and Spark SQL were chosen as representatives for batch processing. For stream processing, the superset of StreamBench and workloads related to real-time streaming analytics were selected. They claim that if the only difference between batch and stream is micro-batching, the batch and stream workloads will have the same micro-architectural level behaviours. They also claim that Spark workloads with DataFrames have better micro-architectural performance than RDD.

Mishra et al. [34] proposed a method to classify workloads and apply it to Google compute clusters. The authors claim that coarse-grain and fine-grain are not sufficient to classify big data workloads. They proposed three metrics (task duration, CPU usage, memory usage) with three qualitative values (small, medium and large) to represent resource utilisation of tasks. While the three metrics were used as input data features, k-means was used to do classification. Their method focused on system-level metrics. 


\subsection{Limitations of Previous Research}

1. Most of the research focuses on automatic tuning of either Hadoop only or Spark only. Since there are many analytics frameworks in use, it is necessary to have a tuning system that can apply to several frameworks.

2. The data size of these previous research experiments is on the GB level which is far from scenarios faced in industry. The conclusions from small data size experiments might not accurately reflect the performance of big data size applications.

3. In these experiments, the applications are simple single workloads which are different from the situation in industry. It is very uncommon for just one user to run a single workload as an application on Hadoop or Spark. Multi-user and multi-workload are more common cases. However, these research teams did not try to simulate these kinds of scenarios.

4. All the tuning paper provide the tuning method, but none of them mention when the tuning method should be invoked. This leads to a tuning overhead.

5. Only a few research teams have analysed the characteristic of Hadoop and Spark workloads. And none of the research done by these teams focused on detecting workload transitions inside of a job flow on the container level. 


\section{Section 4}

\section{Overview of Kermit}

Kermit is the first on-line automatic tuning system for YARN [1]. Kermit was released before I joined the team. The published paper "Automatic, on-line tuning of YARN container memory and CPU parameters" [1] provides more details about Kermit. Based on this paper [1], the design of Kermit, and inspiration from Kermit will be introduced in this Section.

\subsection{Design of Kermit}

Comprised of AutoTuner and Analyzer, Kermit was implemented using Java and integrated with YARN. It is a generic automatic tuning system which can work with any YARN-based analytics framework, for example, Hadoop(2011) and Spark(2014). Figure 4.1 shows how Kermit works together with frameworks.

\subsubsection{How Kermit Improves the Performance of Frameworks}

Each job running on Hadoop or Spark consists of many tasks, and every task runs inside of a container. The amount of available resources for the current job divided by the number of resource requests for each container determines the number of containers. Since the amount of available resource is fixed, too many containers running at the same time means each container has fewer resources which leads to a longer time to finish each task. Too few containers running at the same time means the resources provided by the cluster are not fully utilized. Neither too many 


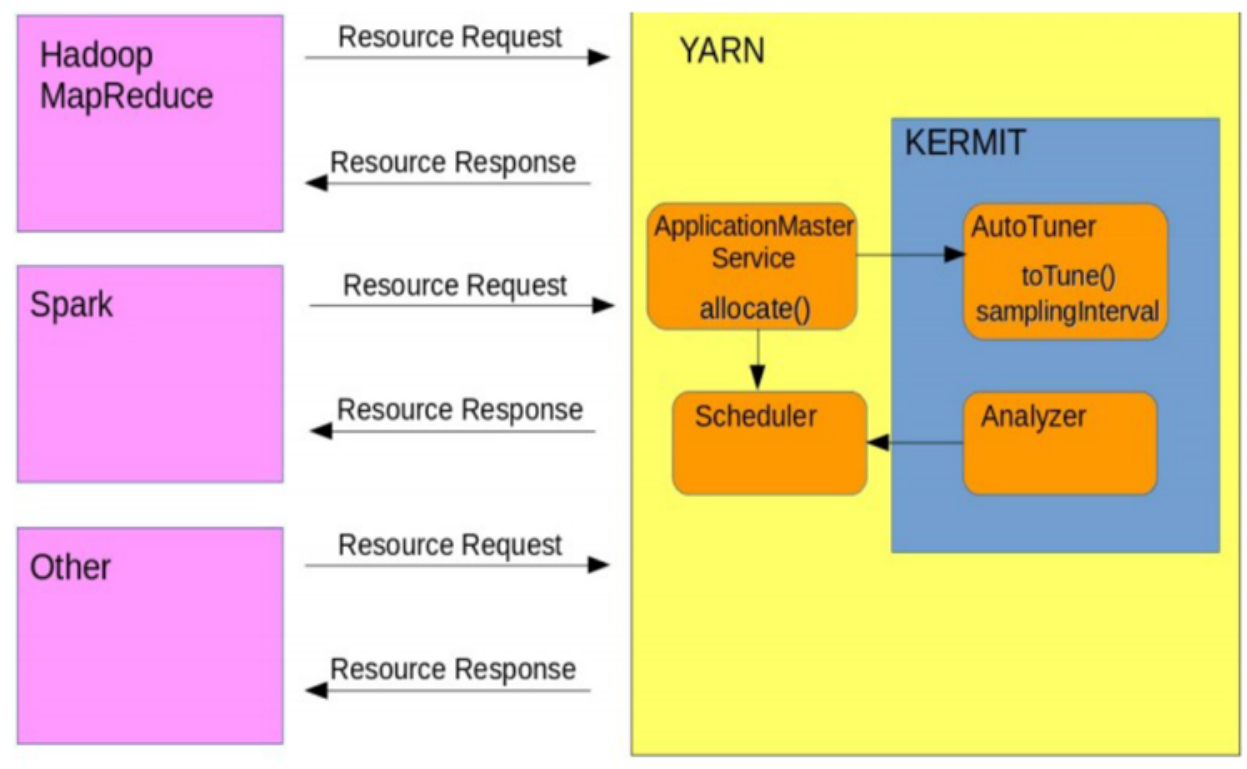

Figure 4.1: KERMIT architecture. From [1]

nor too few containers running simultaneously leads to the best performance of the framework. If the automatic tuning system can choose an appropriate number of containers to run synchronously, the performance of the framework will be improved. In order to get an optimal number of concurrent running containers, the parameters related to request resources should be assigned optimal values. This research focused on two kinds of resources: CPU and memory. The six parameters that determine the amount of CPU and memory in each Hadoop container or each Spark executor are as follows:

1. mapreduce.map.memory.mb: The amount of memory to request from the scheduler for each map task [36]

2. mapreduce.map.cpu.vcores: The number of virtual cores to request from the scheduler for each map task [36]

3. mapreduce.reduce.memory.mb: The amount of memory to request from the scheduler for each reduce task [36]

4. mapreduce.reduce.cpu.vcores: The number of virtual cores to request from the scheduler for each reduce task [36]

5. spark.executor.memory: Amount of memory to use per executor process [37] 


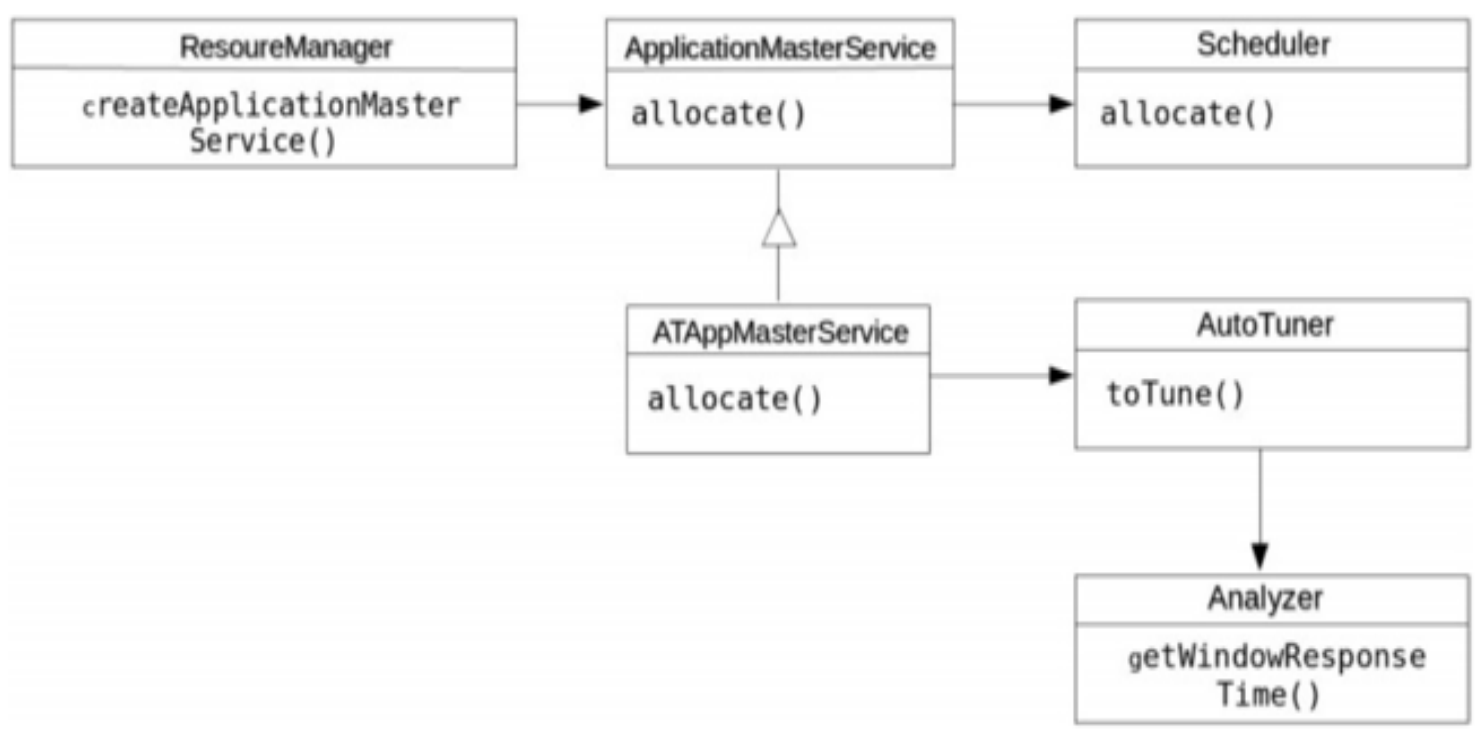

Figure 4.2: How KERMIT integrates with YARN. From [1]

6. spark.executor.cores: The number of cores to use on each executor [37]

In summary, by assigning the optimal values to the above six parameters, Kermit runs the optimal number of containers simultaneously on Hadoop and Spark which leads to improving the overall performance of the frameworks.

\subsubsection{Integration Kermit with YARN}

Figure 4.2 shows the integration of Kermit and YARN. The allocate() function, which is used to send resource requests from ApplicationMaster to ApplicationMaster Service, was designed to be overridden by Kermit. The AutoTuner component leverages the Explorer algorithm to search for the optimal values of the parameters, modifying the amount of requested CPU and memory. After setting those parameters, Analyzer will observe the effect of the new values by calculating the average and standard deviation of container completion time and then provides feedback to the Explorer algorithm. 


\subsection{Inspiration From Kermit}

After evaluation, compared to the results from the best possible tuning strategy, Kermit was able to achieve $92 \%$ efficiency [1] over the basic tuning. However, Kermit still has the problem of tuning overhead especially in executing complex jobs. In order to reduce tuning overhead, the tuning method should be evoked after the transition has happened. This means that the workload characteristics have changed. If a tuning method is evoked after the transition, the tuning overheard will be decreased and the performance of Kermit for complex jobs will be improved. This idea motivated us to consider how to detect workload transitions and inspired the research work of this thesis. 


\section{Section 5}

\section{Experimental Design and Implementation}

In this section, twenty-six experiments and related concepts will be introduced. The aim of these experiments is to collect container performance metrics data for different kinds of workloads.

\section{$5.1 \quad$ Design of Experiments}

\subsubsection{Related Concepts}

The experiment was designed as the first experimental study of container performance metrics observed in Hadoop and Spark workloads. In order to explain these metrics more clearly, the two basic concepts used will be introduced in this subsection.

\subsubsection{Related Concepts}

\section{A. Container}

A container refers to the constructor for memory, CPU, disk space, etc. These are allocated by the resource manager. Hadoop and Spark send resource requirements to the resource manager, and then their tasks will be run inside of containers. Even if different frameworks use containers in different ways, they depend on the container to finish their job. For example, while the Hadoop task runs in the container, the Spark executor also runs in the container. So, observing containers performance can also reflect the performance of the framework which explains the reason for our experimental study on container performance metrics. 


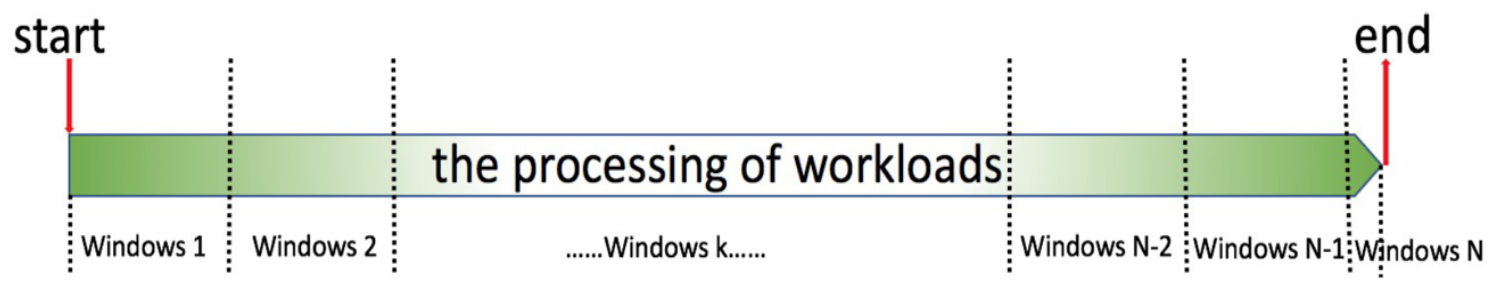

Figure 5.1: Windows

\section{B. Window}

Windows are used to divide the execution process of workloads. It can be simply understood as a time interval. One workload will have many windows in sequence, and every window has a consecutive ID number. In our experiments, data was collected by each window as shown in Figure 5.1

\subsubsection{Container performance metrics}

In our study, we focused on four kinds of container performance metrics: container creation rate, container completion rate, container average response-time, container response-time relative standard deviation(RSD). For each metric, we gave a definition, steady-status value, and dynamic value.

\section{A. Container Creation Rate(Ctr)}

This metric is related to the new created container in the observed window. Ns(ctr) is equal to the number of containers started in the observation window n. Steady status value (Ctr) is equal to $\mathrm{Ns}(\mathrm{n}) /$ (window's duration in minute), which normalizes the number of containers created in the window $\mathrm{n}$ to one minute. Dynamic value is equal to the steady status value in window $n$ divided by the steady status value in the window(n-1). This value reflects the changeability between two adjacent windows.

\section{B. Container Completion Rate(Cplt)}

This metric is related to the containers that finished execution in the observed window. $\mathrm{Nf}(\mathrm{n})$ can be defined as the number of containers finished in the observation window n. Steady status value (Cplt) equals $\mathrm{Nf}(\mathrm{n}) /($ window's duration in minute), which normalizes the number of containers finished in window $n$ to one minute. Dynamic 
value equals to steady status value(Cplt(n)) in window $\mathrm{n}$ divided by steady status value $(\mathrm{Cplt}(\mathrm{n}-1))$ in window(n-1). This value reflects the changeability between two adjacent windows.

\section{Container Average Response Time(Rt)}

This metric relates to the average response time for all containers in the observed window. Rti can be defined as the response time of container $i$ in the observation window n. Steady status value (Rt) equals to $\operatorname{Sum}(\mathrm{Rti}) \mathrm{n} / \mathrm{Nf}(\mathrm{n})$ which calculates the average container response time in window $\mathrm{n}$. Dynamic value equals steady status value $(\operatorname{Rt}(\mathrm{n}))$ in window $\mathrm{n}$ divided by steady status value $(\mathrm{Rt}(\mathrm{n}-1))$ in window(n-1). This value reflects the changeability between two adjacent windows.

\section{Container Response-time Relative Standard Deviation(RSD)}

This metric shows the scatter degree of containers response time in the observed window. Steady status value (RSD) equals $\operatorname{Sum}(\mathrm{Rti}) \mathrm{n} / \mathrm{Nf}(\mathrm{n})$ which calculates the average container response time in window $n$ divided by the standard deviation of average containers response time in the observation window n. Dynamic value equals to steady status value $(\operatorname{RSD}(\mathrm{n}))$ in window n divided by steady status value $(\operatorname{RSD}(\mathrm{n}-1)$ ) in window(n-1). This value reflects the changeability between two adjacent windows.

\subsubsection{The Goal of Experiments}

The twenty-six experiments were designed to run single workload, workloads in sequence, and interactive workloads using common big data benchmarks. Four kinds of container performance metrics were calculated from the execution logs. Based on the collected data, the aim was to determine whether these metrics can identify the workload type and detect workload change. At the same time, experiments were run on Hadoop and Spark in order to prove that our results can apply to more than one kind of analytics frameworks. 


\subsection{The Implementation of Experiments}

\subsubsection{Cluster Setup}

All experiments were running on an eight-node cluster which comprised of one master node and seven worker nodes. Figure 5.2 summarizes the cluster architecture. These nodes were running on Virtual Machines(VM). Table 5.1 shows the configuration of VM in details. The configuration of host VM is shown on Table 5.2. A 100 GB SSD drive was used to serve every node. Each drive contained a Hadoop and Spark installation, as well as the operating system. All the nodes were connected by a 10GB fiber switch and had access to a shared 12TB Network Storage SSD Driver.

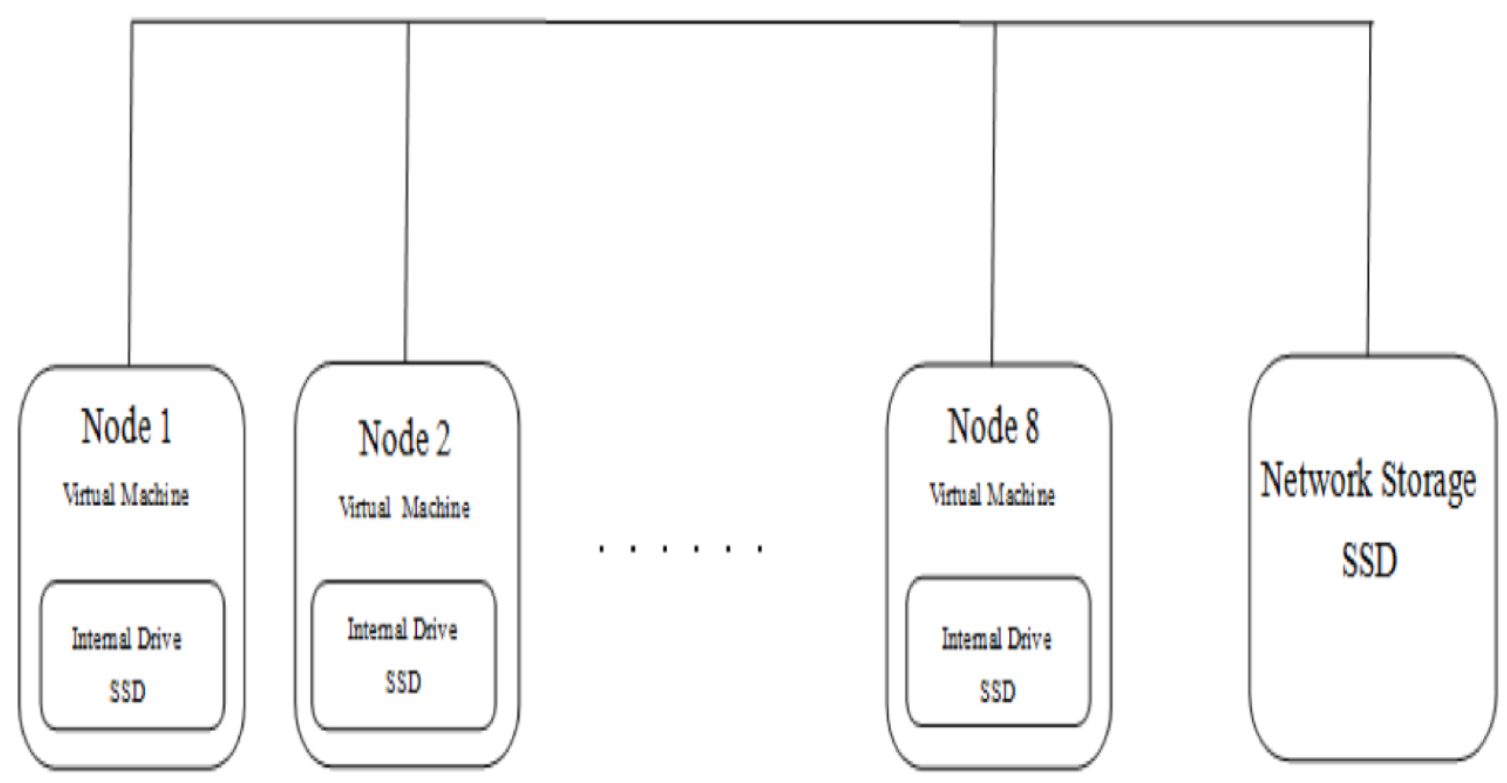

Figure 5.2: Cluster architecture. From [2] 


\begin{tabular}{|c|c|}
\hline Component & Parameter \\
\hline Number of VMs & 8 \\
Operation System & Ubuntu 16.04 \\
Memory & $48 \mathrm{~GB}$ RAM \\
CPU & 10 vCPU \\
Storage Size & $100 \mathrm{~GB}$ SSD \\
\hline
\end{tabular}

Table 5.1: Configuration of host VM.

\begin{tabular}{|c|c|}
\hline Component & Parameter \\
\hline Operation System & Ubuntu 14.04 \\
Core & Dual 10-core \\
Memory & 128GB DDR3 RAM \\
CPU & IBM S822L Power8 \\
Hard Disk Drive for OS & 146GB 15K SAS HDD \\
Hard Disk Drive for Local Disk & $3 * 1.2 \mathrm{~TB}$ 10K RPM SAS HDDs \\
\hline
\end{tabular}

Table 5.2: Configuration of cluster nodes.

\subsubsection{Parameters Setting}

In our study, experiments were run on Hadoop2.7 and Spark 2.1.1. Values were assigned to the parameters in Table 5.3, in order to avoid running out of space and decreasing the possibility of the bottleneck for container performance. Only the parameters mentioned in Table 5.3 were modified, other parameters in the Hadoop and Spark were kept as their default values. 


\subsubsection{Benchmarks}

Various benchmarks were used to simulate Hadoop and Spark workloads and workload transitions. These benchmarks include HiBench, TeraSort suite, SparkMultiuserBenchmark2(SMB2), and spark-perf. The reason that these benchmark are used in our research is that these are popular and used frequently in the automatic automatic tuning field.

Table 5.4 lists the benchmarks running on Hadoop and Spark in this experiments, respectively.

\begin{tabular}{|r|c|}
\hline yarn.nodemanager.resource.cpu-vcores & the total number of CPUs in the node \\
\cline { 1 - 2 } yarn.nodemanager.resource.memory-mb & \multirow{2}{*}{ total amount of memory in the node } \\
\cline { 1 - 2 } yarn.scheduler.maximum-allocation-mb & \multirow{2}{*}{36} \\
\hline mapreduce.job.reduces & \multirow{2}{*}{ TRUE } \\
\hline mapreduce.output.fileoutputformat.compress & org.apache.hadoop.io.compress \\
\hline mapreduce.map.output.compress & 850mb \\
\hline mapreduce.output.fileoutputformat.compress.code & $6 \mathrm{G}$ \\
\hline mapred.child.java.opts & \\
\hline
\end{tabular}

Table 5.3: Assign values to parameters. 


\begin{tabular}{ll}
\hline \hline \multicolumn{1}{c}{ Category } & \multicolumn{1}{c}{ Workload } \\
\hline Micro Benchmarks & Sort \\
& WordCount \\
& TeraSort \\
& EnhancedDFSIO \\
\hline Web Search & Nutch Indexing \\
& Page Rank \\
\hline Machine Learning & Bayesian Classification \\
& K-means Clustering \\
\hline Analytical Query & Hive Join \\
& Hive Aggregation \\
\hline \hline
\end{tabular}

Figure 5.3: Cluster architecture. From [3]

\begin{tabular}{|c|c|c|}
\hline Benchmark & Run on Hadoop & Run on Spark \\
\hline HiBench-WordCount, Kmeans & Y & N \\
Terasort/HiBench-Terasort, TeraGen, TeraValidate & Y & Y \\
SMB2 & N & Y \\
Spark-perf & N & Y \\
\hline
\end{tabular}

Table 5.4: Benchmark in the experiments

\section{A. HiBench}

HiBench is provided by Intel and has been widely used to evaluate and tune Hadoop. Since it can provide real-world applications for Hadoop, users can observe the character and behavior of Hadoop applications [3]. HiBench consists of 4 categories with ten workloads which are synthetic and representative for Hadoop. The details are shown in Figure 5.3 
Since different configurations represent different workloads, we can modify parameters in the Hibench configuration file [38] to get different workloads. We can set a coarse-grain data scale parameter for all sub-benchmarks in Hibench by modifying the HiBench/blob/6.0/conf/hibench.conf file. In this file, $\mathrm{Hi}$ bench.scale.profile is the parameter for data scale which can be selected as tiny, small, large, huge, gigantic or bigdata. While hibench.default.map.parallelism defines the number of mappers in MapReduce, hibench.default.shuffle.parallelism defines the number of reducers in MapReduce. For each sub-benchmark, we can assign numeric values to a data scale. For example, in wordcount, we can open the HiBench/blob/master/conf/workloads/micro/wordcount.conf file and assign different values to data scales according to different experiments requirements.

\section{B. TeraSort Suite}

Terasort Suite is a common Hadoop benchmark which was proposed by Yahoo [39]. It consists of TeraGen, TeraSort, and TeraValidate [40].

\section{TeraGen}

TeraGen is used for generating the required size of data which is the input for TeraSort. The command for executing TeraGen in the Hadoop home directory is:

hadoop jar /usr/local/hadoop/share/hadoop/mapreduce/hadoop-mapreduceexamples-2.7.3.jar teragen 10000000000 outputfilepath

The first parameter denotes the number of rows in the generation data file, and each row has 100 bytes. The data size in MB equals the first parameter divided by 10. Each row is composed of three parts: keys, rowid, and filler. The details are shown in Figure 5.4.

\begin{tabular}{|l|l|l|l|l|}
\hline 10 bytes key & 10 bytes rowid & 78 bytes filler & Ir & In \\
\hline
\end{tabular}

Figure 5.4: The component of each row in TeraGen.

TeraSort

TeraSort is a MapReduce program which results in globally sorted output data. If 
the number of reducers is $\mathrm{N}$, a sorted array of $(\mathrm{N}-1)$ sample keys from the input data will be created. For reducer i, it only receives data with keys that satisfy [sample[i-1], sample[i]]. In this way, the process can guarantee that output in the reducer $\mathrm{i}$ is greater than the output in the reducer (i-1). Figure 5.5 is an example of this process.

\section{Sample key array $\{3,15, \ldots ., 89\}$}

\section{Keys in reducers $0,1,2,3$ Reducer1 Reducer2

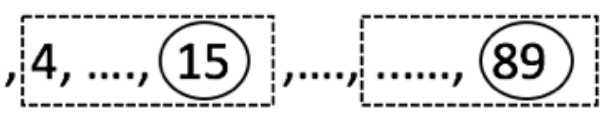 \\ Reducer3

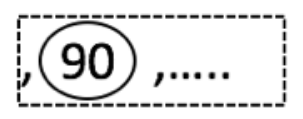 \\ Reducer4}

Figure 5.5: MapReduce process in TeraSort.

The command for executing TeraSort is as follows:

hadoop jar /usr/local/hadoop/share/hadoop/mapreduce/hadoop-mapreduceexamples-2.7.3.jar terasort inputfilepath outputfilepath

TeraValidate

TeraValidate is the next step after TeraSort which is used for validating the output of TeraSort. If the input data isn't globally sorted, the output file records the key. Otherwise, the output file is empty. The command for executing TeraSort is as follows: hadoop jar/usr/local/hadoop/share/hadoop/mapreduce/hadoop-mapreduceexamples-2.7.3.jar teravalidate inputfilepath outputfilepath

\section{Spark-perf}

Spark-perf, released by Databricks, is a common benchmark for Spark. The benchmark suites include the performance test for Spark, PySpark, SparkStreaming and Mlib [41]. In the configuration file, users can control which kinds of workloads will be running by modifying the value for RUN_benchmarkName and PREP_bechmarkName. Compared with Hibench and Terasort, the data size of sparkperf is fixed. Users can modify the numeric value of SCALE_FACTOR to assign the different size of input data to the benchmark workloads.

\section{SparkMultiuserBenchmark2(SMB2)}

SMB2 was released by IBM. It can simulate multiusers to run workloads concurrently 
and sequentially [42]. Hadoop creates the container for each task and recycles it when it is done. Spark executors run as containers and executors typically are static allocations which means the running time of executors are the same with the Spark application lifetime. As a result, it is necessary to choose a benchmark according to the characteristics of Spark. SMB2 is composed of 4 user cases:

1. User case 1-Synch-interactive multi-user

2. User case 2-Asynch-batch multi-user

3. User case 3-Mixed multi-user

4. User case 4-Mixed multi-tenant

These four cases are based on synchronous interactive workloads and asynchronous batch workloads. In our study, we ran experiments on user case 3. In this case, multiuser can run synch-interactive and asynch-batch workloads. Since section 6 provides data visualization of user case1 and user case 3, they will be explained in more detail in section 6 .

\subsubsection{Experimental Procedures}

These are seven steps we followed to execute our experiments.

1. The first step is to start Hadoop and Spark.

2. Second, we start nmon, which is used for collecting hardware performance data, such as, the utilization of CPU and memory.

3. Third, benchmarks present in Figure 5.6 are run.

4. After all workloads in benchmarks finished, nmon stopped in the fourth step.

5. Next, nmon data are collected and Kermit.log is generated which records the information about containers and windows.

6. Since the data collected through Kermit.log is not very readable, the sixth step is utilizing JAVA code to transfer valuable data in Kermit.log file into the container.csv file. The container id, corresponding starting window id, and finishing window id are recorded in the container.csv file. 
7. After ensuring the nmon data file and container.csv are saved, the last step is to clear the other files in the experiment.

\begin{tabular}{|c|c|c|}
\hline Transition & Description & Benchmarks, Procedure and Data Size \\
\hline Hd-sj-1 & Transition from map to reduce processing in a single Hadoop map-reduce job. & "HiBench WordCount benchmark. 2 TB. \\
\hline Hd-sj-2 & Transition from map to reduce processing in a single Hadoop map-reduce job. & TeraSort benchmark. 2 TB. \\
\hline Hd-sj-3 & $\begin{array}{l}\text { Transition from reduce-shuffle to reduce processing in a single Hadoop map- } \\
\text { reduce job. }\end{array}$ & TeraSort. 2 TB. \\
\hline Hd-sufl-4 & $\begin{array}{l}\text { Transition from TeraGen to TeraSort processing in a Hadoop single-user job } \\
\text { flow. }\end{array}$ & $\begin{array}{l}\text { TeraGen-TeraSort-TeraValidate sequence of jobs. } 2 \\
\text { TB. }\end{array}$ \\
\hline Hd-sufl-5 & $\begin{array}{l}\text { Transition from TeraSort to TeraValidate processing in Hadoop single-user job } \\
\text { flow. }\end{array}$ & $\begin{array}{l}\text { TeraGen-TeraSort-TeraValidate sequence of jobs. } 2 \\
\text { TB. }\end{array}$ \\
\hline Hd-sj-6 & $\begin{array}{l}\text { Transition from one iteration to another within Hadoop K-Means machine } \\
\text { learning job. }\end{array}$ & HiBench K-Means. 2 TB. \\
\hline Hd-sufl-7 & $\begin{array}{l}\text { Transition from Hadoop WordCount reduce processing to TeraSort map pro- } \\
\text { cessing in a single-user job flow. }\end{array}$ & $\begin{array}{l}\text { HiBench WordCount-TeraSort-K-Means job flow. } 2 \\
\text { TB. }\end{array}$ \\
\hline Hd-sufl-8 & $\begin{array}{l}\text { Transition from TeraSort reduce processing to K-Means processing in a single- } \\
\text { user job flow. }\end{array}$ & $\begin{array}{l}\text { HiBench WordCount-TeraSort-K-Means job flow. } 2 \\
\text { TB. }\end{array}$ \\
\hline Hd-mufl-9 & Multi-user transition from TeraSort shuffle to K-Means. & 2 users (1 running TeraSort, and $1 \mathrm{~K}-$ Means) 2 TB. \\
\hline Hd-mufl-10 & Multi-user transition from K-Means iteration back to TeraSort reduce phase. & 2 users (1 running TeraSort, and $1 \mathrm{~K}$-Means) $2 \mathrm{~TB}$. \\
\hline Hd-mufl-11 & Multi-user transition from TeraSort map phase to K-Means iteration. & 2 users (1 running TeraSort, and $1 \mathrm{~K}-$ Means) $2 \mathrm{~TB}$. \\
\hline Hd-mufl-12 & Multi-user transition from K-Means iteration to TeraSort map phase. & 2 users (1 running TeraSort, and $1 \mathrm{~K}$-Means) $2 \mathrm{~TB}$. \\
\hline Hd-mufl-13 & Multi-user transition from TeraSort map phase to K-Means iteration. & 3 users (1 running TeraSort, and 2 K-Means) 2 TB. \\
\hline Hd-mufl-14 & Multi-user transition from K-Means iteration to TeraSort reduce phase. & 3 users (1 running TeraSort, and $2 \mathrm{~K}$-Means) $2 \mathrm{~TB}$. \\
\hline Sp-sj-1 & Transition from map() to reduceByKey() processing in a single Spark job. & Spark ARL TeraSort. 2 TB \\
\hline Sp-sufl-2 & Transition from Spark K-Means processing to TPC-DS-inspired Q3. & $\begin{array}{l}\text { SMB-2 } 1 \text { user, use case } 2 \text { (batch analytics) Spark job } \\
\text { sequence. } 2 \text { GB per application. }\end{array}$ \\
\hline Sp-sufl-3 & Transition from Spark TPC-DS-inspired Q3 to Q53. & $\begin{array}{l}\text { SMB-2 } 1 \text { user, use case } 2 \text { (batch analytics) Spark job } \\
\text { sequence. } 2 \text { GB per application. }\end{array}$ \\
\hline Sp-sufl-4 & Transition from Spark TPC-DS-inspired Q53 to Q89. & $\begin{array}{l}\text { SMB-2 } 1 \text { user, use case } 2 \text { (batch analytics) Spark job } \\
\text { sequence. } 2 \text { GB per application. }\end{array}$ \\
\hline Sp-sufl-5 & Transition from Spark TPC-DS-inspired Q89 to Q8. & $\begin{array}{l}\text { SMB-2 } 1 \text { user, use case } 2 \text { (batch analytics) Spark job } \\
\text { sequence. } 2 \text { GB per application. }\end{array}$ \\
\hline Sp-mufl-6 & $\begin{array}{l}\text { Transition from Spark single-user batch processing to multi-user ( } 3 \text { interactive } \\
\text { users). }\end{array}$ & $\begin{array}{l}\text { SMB-2 } 1 \text { batch user }+3 \text { interactive users, use case } \\
3 \text { (mixed analytics) Spark job sequence. } 2 \text { GB per } \\
\text { application. }\end{array}$ \\
\hline Sp-mufl-7 & $\begin{array}{l}\text { Transition from Spark multi-user ( } 3 \text { interactive users) to single-user batch } \\
\text { processing. }\end{array}$ & $\begin{array}{l}\text { SMB-2 } 1 \text { batch user }+3 \text { interactive users, use case } \\
3 \text { (mixed analytics) Spark job sequence. } 2 \text { GB per } \\
\text { application. }\end{array}$ \\
\hline Sp-sufl-8 & Initiation of Spark streaming. & spark-perf benchmarking suite. \\
\hline Sp-sufl-9 & Completion of Spark streaming. & spark-perf benchmarking suite. \\
\hline Sp-sufl-10 & Transition from Spark aggregateByKey to aggregateByKey(Int). & spark-perf benchmarking suite, data scale 3 . \\
\hline Sp-sufl-11 & Transition from Spark aggregateByKey to sortByKey(). & spark-perf benchmarking suite, data scale 3 . \\
\hline Sp-sufl-11 & Transition from Spark count() to filter(). & spark-perf benchmarking suite, data scale 3 . \\
\hline
\end{tabular}

Figure 5.6: Twenty six experiments.From [2]

\subsubsection{Experimental Data}

By leveraging the four kinds of benchmarks, the experiments were designed in an increasing order of complexity. Figure 5.6 shows the detail of the experiments. The description explains which type of workloads is running in the current transition 
while the last column in the figure denotes the data size.

Hd-sj-1, Hd-sj-2, Hd-sj-3 and Hd-sj-6 capture the transitions inside simple jobs in Hadoop workloads. Transitions inside a Spark single simple job is captured by Sp-sj-1. Then complex single-user job flows (Hd-sufl-4, Hd-sufl-5,Hd-sufl-7, Hd-sufl-8 for Hadoop and Sp-sufl-2, Sp-sufl-3, Sp-sufl-4, Sp-sufl-5, Sp-sufl-8 to Sp-sufl-10 for Spark) are executed to capture workloads transition inside job flows for Spark. Finally, the experiments proceeded to multi-users job flows which are from Hd-mufl-9 to Hd-mufl-14 for Hadoop workloads and Sp-mufl-6,Sp-mufl-7 for Spark workloads. 


\section{Section 6}

\section{Data Analysis and Validation}

\subsection{Data Visualization}

Based on the data collected from the experiments, the steady-state values of the four container performance metrics were calculated. Since these metrics are multi-variate time-series data, data visualization was used to show them and explore whether the four container performance metrics are qualitatively related to workloads patterns and workload transitions.

In every data visualization figure, the horizontal axis tracks the number of observation windows while the vertical axis records the normalized value of each container performance metric. The start point on the horizontal axis is 0 which represents the start of the job flows, and the flowing sequential windows denote the process of executing the job flows. Before running the job, the duration of each window was set as a fixed value.

\subsubsection{Hadoop Single Job}

Figure 6.1 shows the visualization of the WordCount workload in Hadoop. WordCount is used to calculate the frequency of each word in the given file. In the map phase, the workload reads the file, parses it, and generates key-value pairs for each word as output. For the output of this phase, the keys can be replicated while the values are always 1 . The input file of the reduce phase is the output of the map phase. In the reduce phase, the summation is performed for the same key. The output of the reduce phase are key-value pairs while the key is a unique word and the value is 
the number of occurrences of this word.

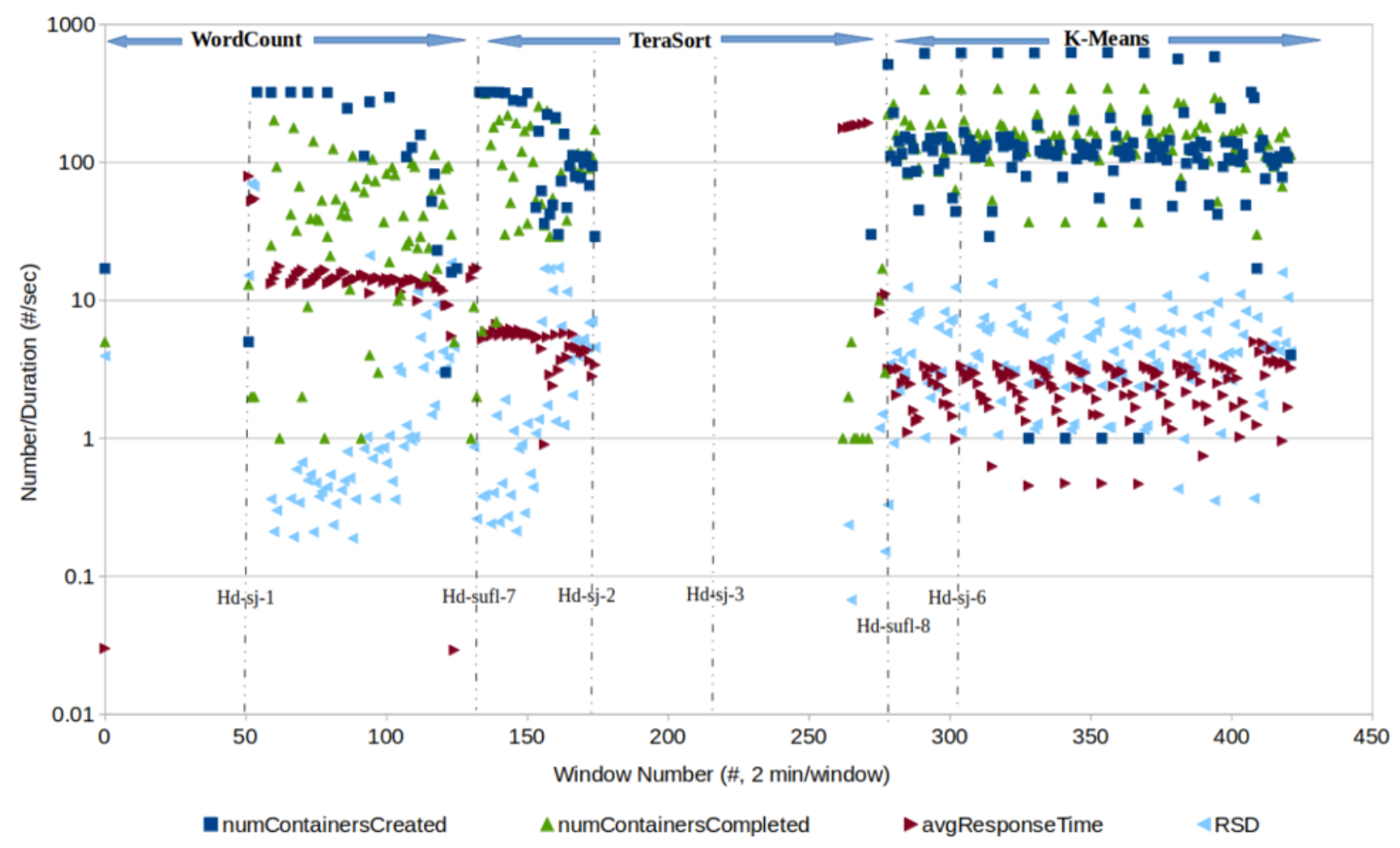

Figure 6.1: Container performance metrics for Hadoop WordCount-TeraSort-KMeans job flows. From [2]

From the Figure 6.1, we can see two different phases clearly inside of WordCount, and the vertical dash line is the boundary for separating different phases. If we observe at the window 0 , the dark blue square shows that some containers are created in the first window. After this activity, the following windows until Window 51 do not show any activity, which means that tasks are running during these windows and each task takes a long time to finish. At Window 51, the green triangle shows the containers are completed around the dashed line which denotes that the map phase is completed. At the same time, we can also observe that the dark blue squares appear again, but they show a different pattern when compared with the map phase. In the Reduce phase, the containers are created and completed continuously which reflects that the reduce tasks need less time to finish when compared with map tasks. The difference between map tasks and reduce tasks leading to the different visualizations, the parsing and generating key-value pairs in the map phase takes more time than the arithmetic summation in the reduce phase tasks. 
If we look at the red triangle which represents the average response time for containers, we can see that the containers finish the map task in about 100 seconds while they finish the reduce task in about 10 seconds. This result also confirms the pattern we observed from container completion rate. When we focus on the light blue triangle which refers to the RSD value of the container, we can see that at the end of the map task, the RSD value is between 10 to 100 while at the start of the reduce task the value is less than 1 . During the reduce phase, the RSD is in an upward trend which is different from the pattern of average response time.

In short, from the data visualization of the WordCount, container performance metrics values are related to the characteristics of the current task and workload transitions in Hadoop.

\subsubsection{Single-user Spark Job Flow}

SMB2-user case 2 refers to multi-user executing asynchronous batch jobs. Multi-user can submit k-means job or TPC-DS-inspired queries jobs without the need to wait for the previous jobs to be completed. The k-means is implemented by Spark MLib while TPC-DS-inspired queries are implemented by Spark SQL. Each job will use its own spark context. Figure 6.2 shows the execution order of this case.

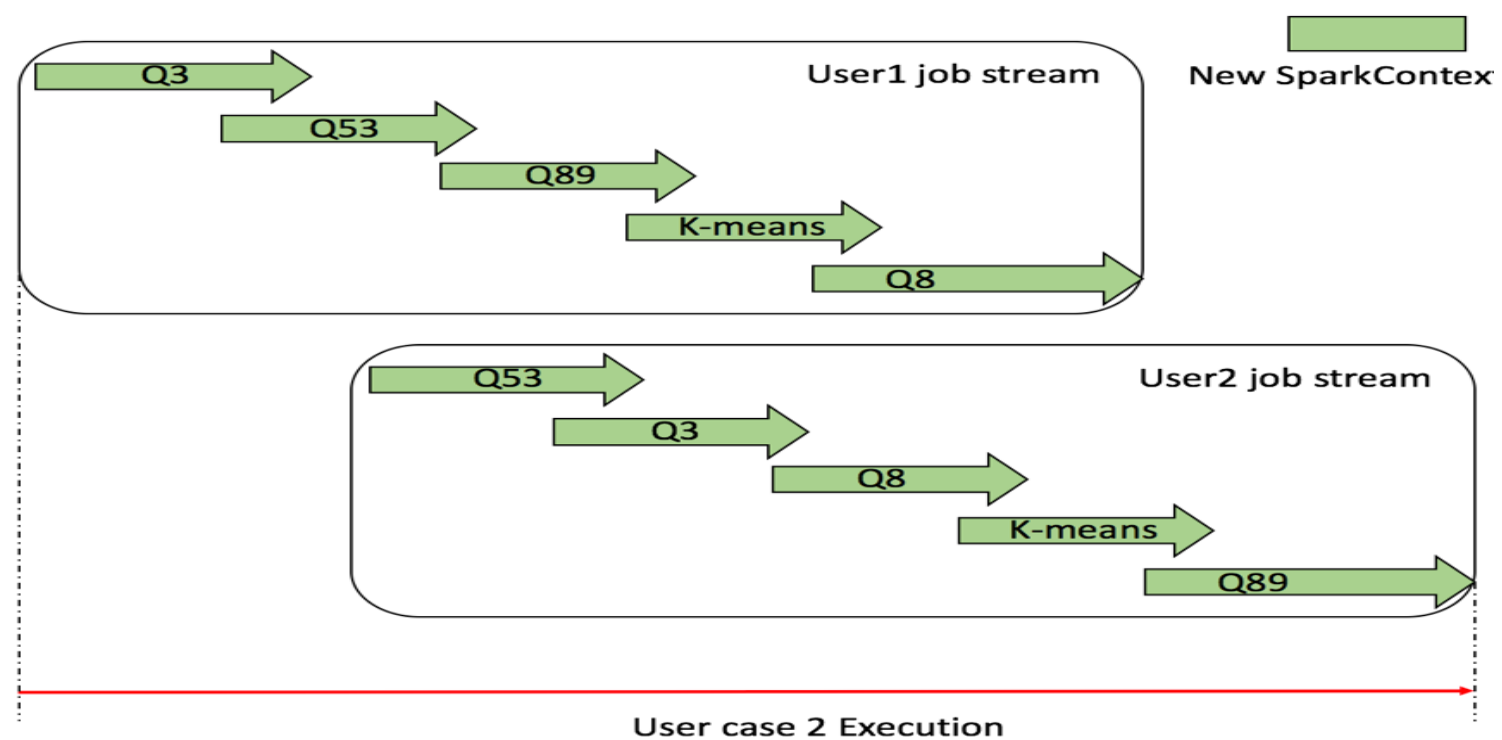

Figure 6.2: Execution order of SMB-userCase2 
Figure 6.3 shows the data visualization of executing SMB2 - user case 2 with a single user. In order to minimize the overlap between jobs, a delay was set between the start of each job. As a result, data was collected from not only sequential jobs but also overlap jobs. The vertical dashed line means that the workload transition happens. At the start of each job, we can observe a sudden burst in the number of container creation. In the following execution of each job, there is no container created. The reason for this pattern is that Spark requests containers only at the start of each job. Since in SMB2-user case 2, each query is submitted to individual SparkContext Figure 6.2, so every query needs its own containers. These containers are requested for executors and Spark re-uses executors to perform the job until the job is completed. After containers were created(the blue square), there is no container activity for a long time. At the end of each job, we can see that there is a sudden burst in the number of container completion(the green triangle), which confirms that most containers will be released at the end of each Spark job. If we look at average response time for containers(the red triangle), it always shows up around the end of each job.

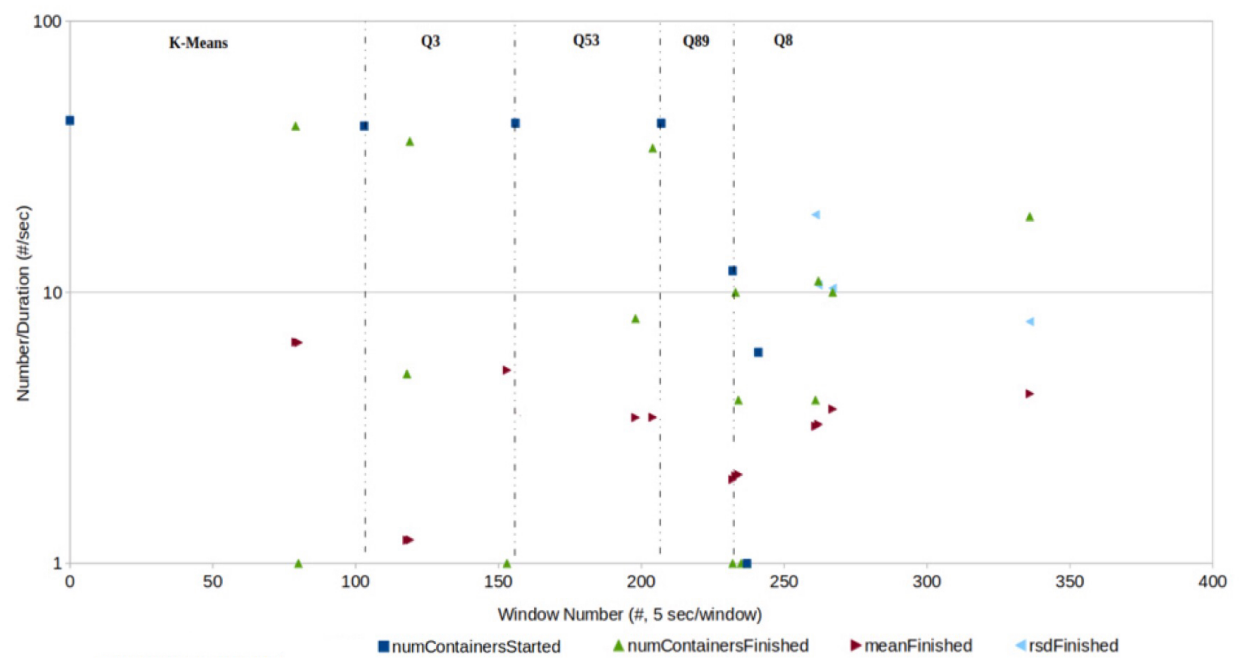

Figure 6.3: Container performance metrics for a sequence of machine-learning and Spark SQL jobs 


\subsubsection{Hadoop Job Flow and Spark Multi-user Job Flow}

Figure 6.1 describes a Hadoop job flow: WordCount-Terasort-K-means. K-means is an iterative algorithm which executes several rounds of a map-reduce job. This job flow simulates a realistic industry data analysis task which is represented by executing MapReduce jobs followed by a machine learning job. From this job flow, we can observe that different jobs show different patterns for the values of the four container performance metrics which means it is possible to detect the workload change according to the change of these metrics' values. If we look at the light blue triangle of WordCount, TeraSort, and each round of K-means map-reduce jobs, we can see they start from low value and increase to higher values as they get close to the completion of the current job. For the red triangle, we can also see an apparent change around the vertical dashed line.

Spark multi-user job flow simulates the SMB2 user case 3 which represents interactive drill-down operations initiated by a human analyst. User case 3 is the combination of user case 2 which is explained in the section 6.1.2 and user case1. SMB2-user case 1 represents multiusers executing synchronous jobs interactively. Before every user submits the next query sequence, they need to wait until the previous query sequences were completed. After the user has submitted the job, SparkContext will be launched, and SparkContext will not be released until one query sequence is completed. These queries run with Spark SQL. Figure 6.4 shows the execution order of this case.

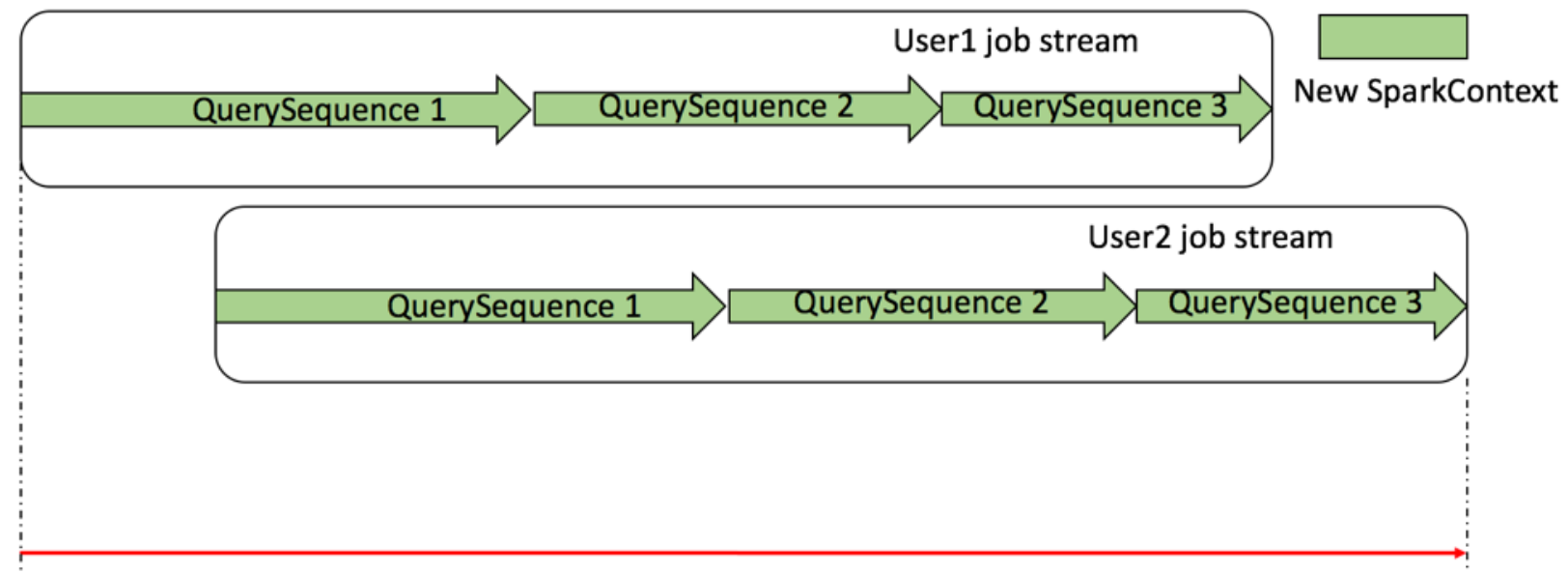

User case 1 Execution

Figure 6.4: Execution order of SMB-userCase1 
In the Spark multi-user job flow experiment, a single user batch synchronous workload (user case2) starts at first. At around Window135, 3 users sync-interactive workloads (user case 1) begin, and each user executes a sequence of TPC-DS-inspired queries. From figure 6.5, we can observe that the values of container performance metrics are different on either side of the vertical dashed lines. Even though this figure does not show which metric changed the most around the vertical dash line, it still illustrates that it is possible to use the change in value of container performance metrics to detect the workload change.

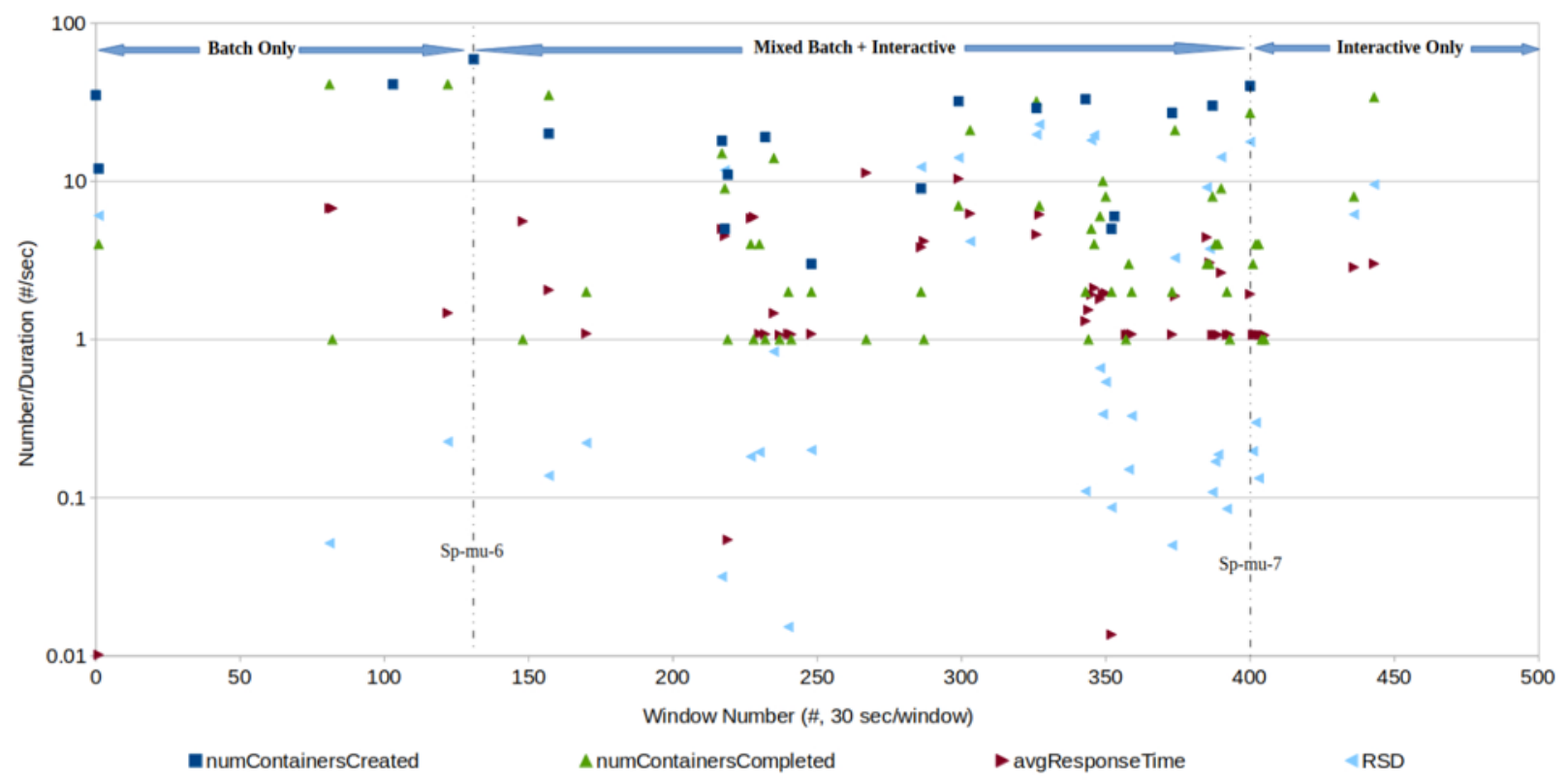

Figure 6.5: Container performance metrics for multi-user Spark workload with batch and interactive query components. From [2]

If we look at Figure 6.1 and 6.5 together, we can see the two figures show fairly different patterns in the number of container creation rate. The allocation of containers in Hadoop workloads happens more frequently than in Spark workloads. It is because Hadoop and Spark use containers in different ways. Since the two data visualization figures show different patterns, it reveals that container performance metrics are possible to distinguish Spark from Hadoop workloads.

In short, from the data visualization of the Hadoop job flow and Spark multiuser job flow(Section 6.1.3), the values of each container performance metric are related to the characteristic of the current workload and workload transitions. 
Above all, the three results of qualitative observations confirm our initial idea that container performance metrics can be used to identify the type of workload and detect workload transitions.

\subsection{Statistical Analysis of Data}

After data visualization, some conclusions can be derived intuitively. However, quantitive analysis is needed to prove that our intuition is correct. Further, some inferential statistical methods were leveraged to get more valuable information from our experimental data.

\subsubsection{The Analysis of Steady State Values and Dynamic Val- ues}

In this part, quantitative methods were performed to analyse the steady state condition and dynamic condition values of the four container performance metrics in order to verify the observations from data visualization.

\subsubsection{The Analysis of Steady State Values}

After collecting all the data of four metrics in every window for Hadoop and Spark workloads, a random sampling was used to select steady state values for analysis. The descriptive statistical methods that are maximum, minimum, average, and standard deviation were applied to all the metrics. After the evaluation, maximum values and average values showed the same trend. Figure 6.6 describes the average value of steady state condition of container performance metrics.

For Hadoop and Spark, they show the same order when we rank the four metrics from the numeric value perspective. The value of container creation rate is the highest while the value of RSD is lowest. The value of completion rate is greater than response-time while it is smaller than creation rate. The most important observation from this figure is that for the four container performance metrics, the values of Hadoop workloads are three times greater than Spark workloads. This 
figure leads to the conclusion that Hadoop and Spark show different patterns in the steady state condition values of container performance metrics.

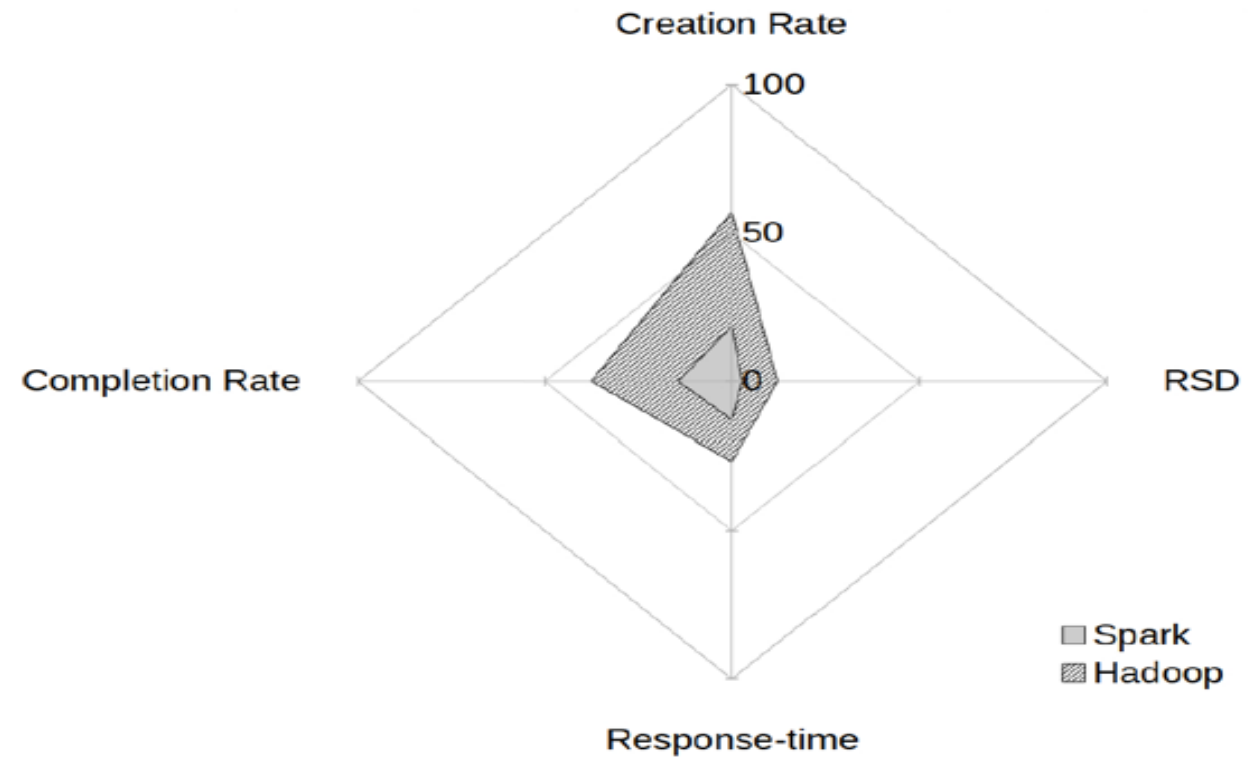

Figure 6.6: Radar chart showing average Hadoop and Spark workload metric values. From [2]

\subsubsection{The Analysis of Dynamic Values}

Since steady state condition values of container performance metrics reveal the different patterns between Hadoop workloads and Spark workloads, we were also interested in how dynamic values perform under similar evaluation. Dynamic value describes the variability of the four container performance metrics before workload transitions and after the transitions. The maximum, minimum, average, and standard deviation of the metrics was calculated. Similar to the steady state analysis, maximum dynamic values and average dynamic values also show the same trend. Figure 6.7 shows the average value of the dynamic condition of container performance metrics. If we look at completion rate and RSD, Hadoop and Spark show a similar trend in the average value of the two metrics. For creation rate and response-time metrics, the average dynamic value of Hadoop is three times greater than Spark. From the figure it can be concluded that Hadoop and Spark show different patterns when the dynamic values which are related to workload transitions are measured. 
Based on the quantitative analysis of steady state value and dynamic value, it can be concluded that it is possible to distinguish Spark from Hadoop workloads by observing the four container metrics.

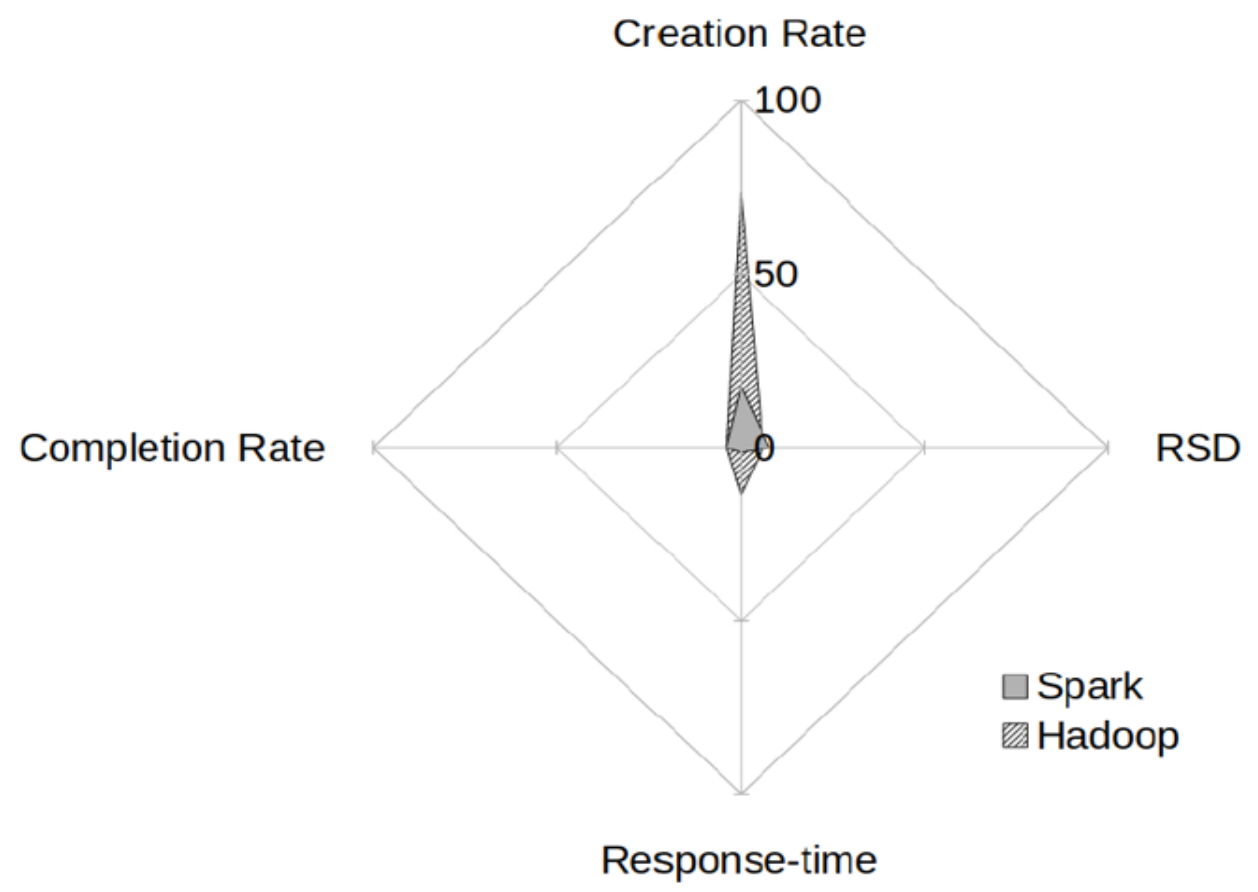

Figure 6.7: Radar chart showing average Hadoop and Spark workload transition values. From [2]

\subsubsection{The Analysis of Correlation Coefficient}

If the container performance metric values before transition equals the value after the transition, it is impossible to use the four metrics to reflect workload transition. In order to prove the metrics are possible to detect the workload change, we need to prove that the values of container performance metrics before workload transitions are different from the values after transitions.

The correlation coefficient is a statistical method to measure whether there is a relationship between two variables [43]. There are three types of correlation 
coefficient: Pearson, Intra-class, and Rank. In our cases, we select Pearson correlation coefficient which is a well-known method to evaluate whether there is a linear relationship between two variables. Our problem transformed into proving the values of container performance metrics before and after workload transitions are not in a linear relationship. We can treat the metrics value before workload transitions as X, after transitions as Y. The flow chart of problem transformation process is shown in Figure 6.8.

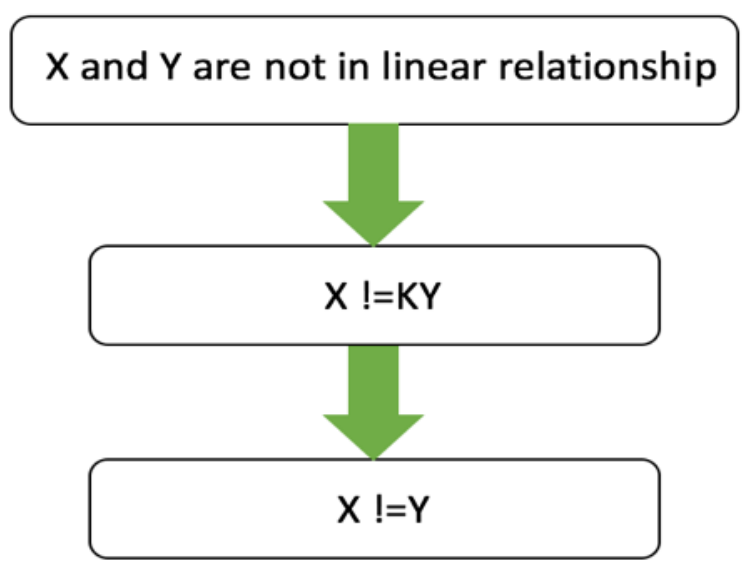

Figure 6.8: Flow chart of problem transformation

The null hypothesis of this problem is that $\mathrm{X}$ and $\mathrm{Y}$ are unrelated. The confidence level for rejecting the null hypothesis is $99 \%$ which is a frequent number in statistical tests. If the $\mathrm{P}$-value is greater than 0.01 , it means that there is not enough evidence to suggest $\mathrm{X}$ and $\mathrm{Y}$ are related at a $99 \%$ confidence level. In other words, at a $99 \%$ confidence level, there is not enough evidence to show the values of container performance metrics before workload transitions is equal to the value after transition. We implemented Python code to do the calculation, and the result is shown in Figure 6.9. From the result, it can be seen that the P-value of container create rate, container duration, and RSD are greater than 0.01 which means that at a $99 \%$ confidence level, there is not enough evidence to show these three metrics values remain the same when workload transitions happen.

So, container create rate, container duration, and RSD can be used to detect the workload change. 
[ [ siyu@osx]Desktop\$./PearsonCorrelation.py

$\begin{array}{lll}\text { Contianer Performance Metrics } & \text { Correlation Value } & \text { P-value } \\ \text { Create Rate Correlation } & 0.2418210159235945 & 0.2339788259709562 \\ \text { Completion Rate Correlation } & 0.5417688129921344 & 0.0042542813282391 \\ \text { Container Duration Correlation } & 0.3020652406785019 & 0.1612608800149824 \\ \text { RSD Correlation } & -0.0145212218115922 & 0.9501862972783286\end{array}$

Figure 6.9: Results of correlation coefficient

\subsection{Data Validation}

Based on the conclusions from section 6.1 and 6.2, machine learning algorithms and Welch's t-test were used to validate that the container performance metrics can be used to classify type of workload and detect workload transitions.

\subsubsection{Identification and Classification of the Workload Type}

In order to validate whether the container performance metrics can classify Hadoop and Spark workloads effectively, machine learning algorithms were leveraged to identify and classify the workload transitions data.

The workload transitions data of container creation rate was collected from two windows before the transitions and two windows after the transitions. According to the type of job flow, these data were labeled as Spark or Hadoop. We randomly split 70 percent workload transition data as training data while the remaining 30 percent was set as testing data. We constructed the classification models by five kinds of machine learning algorithms: K-means, Gradient-Boosted Trees, Logistic Regression, Decision Tree, and Random Forest. For each algorithm, we did the random split 100 times, which means the data size of training is 1820 and testing is 780 , and calculated the average accuracy and standard deviation. This process was implemented by Scala in Spark Mlib. The results are shown in Figure 6.10. 


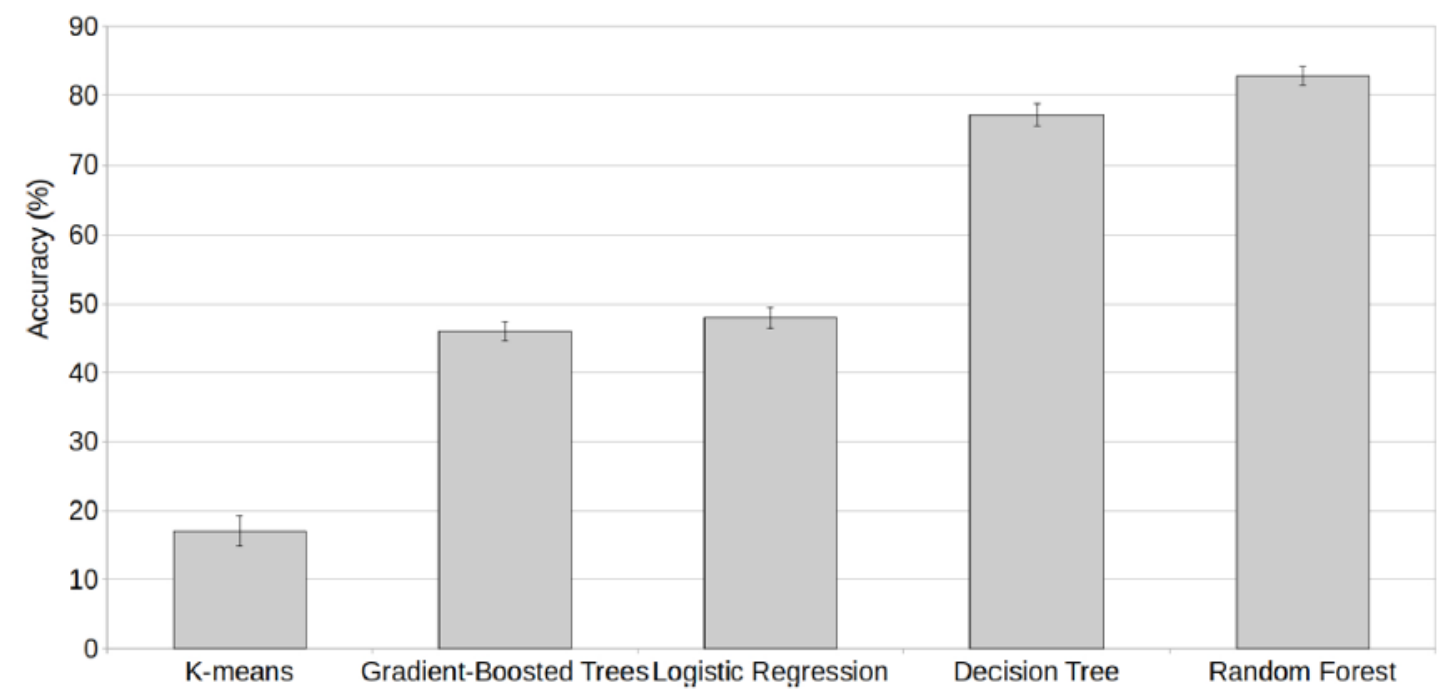

Figure 6.10: Evaluation of classifier

Random Forest algorithm shows the best average accuracy. However, accuracy is not enough to evaluate the performance of a classifier. In order to evaluate whether Random Forest can provide a high performance, we selected one random split and performed the calculation of precision, recall, F1-measure, and ROC curve. The result is shown in Figure 6.11 and Figure 6.12.

In Figure 6.11, 1 is the label for the Hadoop workload while 2 is the label for the Spark workload. F1 score is a measurement to evaluate precision and recall by calculating the harmonic average [44]. The closer the F1 score is to 1, the better performance the classifier has. Figure 6.12 shows the curve of the Receiver Operating Characteristic. The area under this curve reflects the performance of the classifier. Similar to the F1 score, the best value for this area is 1 . From 6.11, we can see the area is 0.87 which means the prediction power of this classifier is strong. 


$\begin{array}{rrrr} & \text { precision } & \text { recall } & \text { f1-score } \\ 1 & 1.00 & 0.80 & 0.89 \\ 2 & 0.75 & 1.00 & 0.86 \\ \text { avg / total } & 0.91 & 0.88 & 0.88\end{array}$

Figure 6.11: Workload classification accuracy for machine-learning algorithms

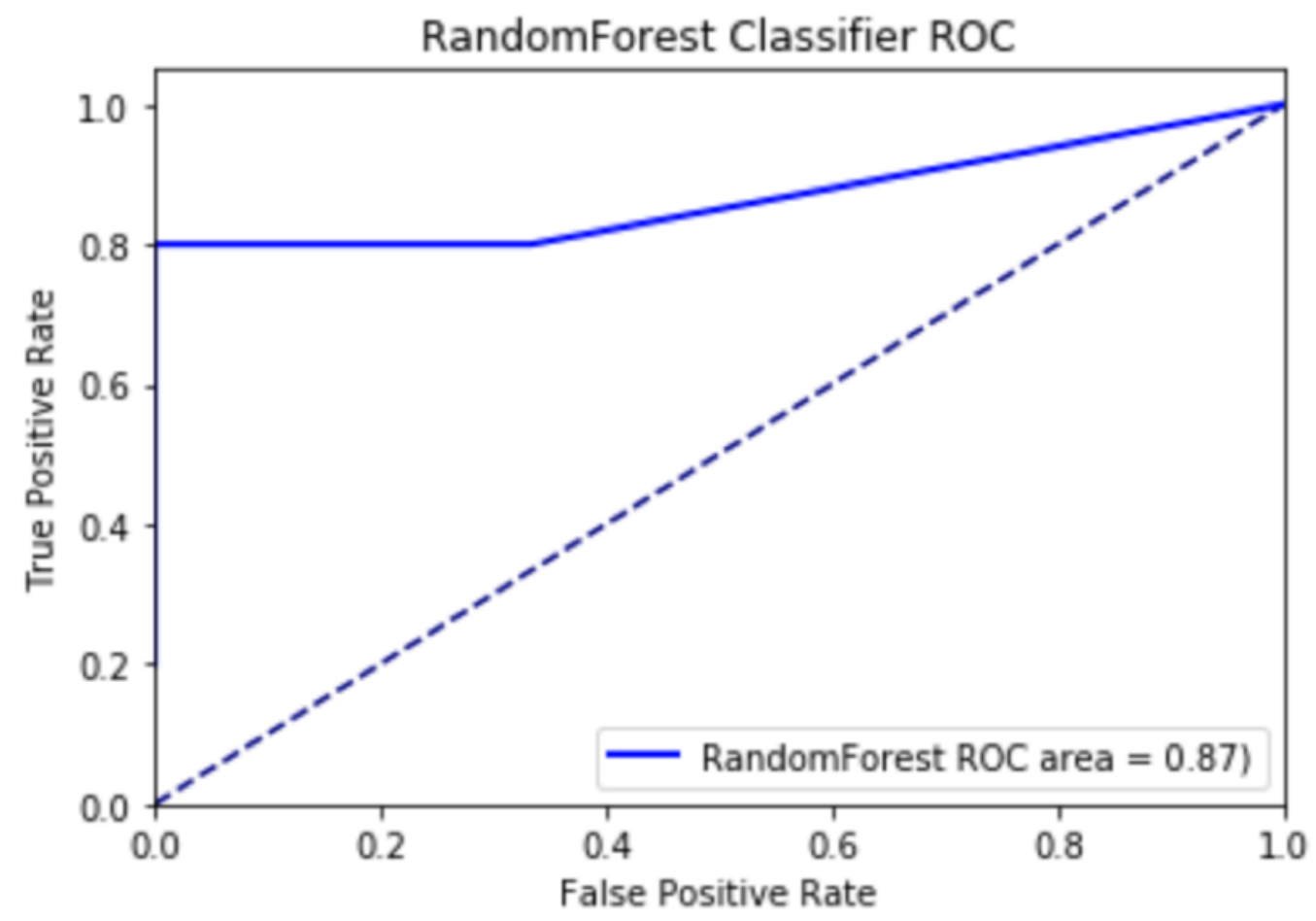

Figure 6.12: ROC

These results illustrate that with container creation rate data only, Random Forest has the highest average accuracy in predicting the workload type, acceptable F1 score, and ROC curve.

In order to observe the effect of different container performance metrics on the accuracy of classifiers, the Random forest algorithm was run on four kinds of input datasets. The details of the input datasets are as follows:

1. Container creation rate only 
2. Container creation rate + Container completion rate

3. Container creation rate + Container completion rate + Average response time

4. Container creation rate + Container completion rate + Average response time $+\mathrm{RSD}$

The results of these four classifiers are shown in Figure 6.13. It can be seen that with the increasing number of features in the input dataset, the average classify accuracy is decreasing. Container creation rate alone provides the highest average accuracy.

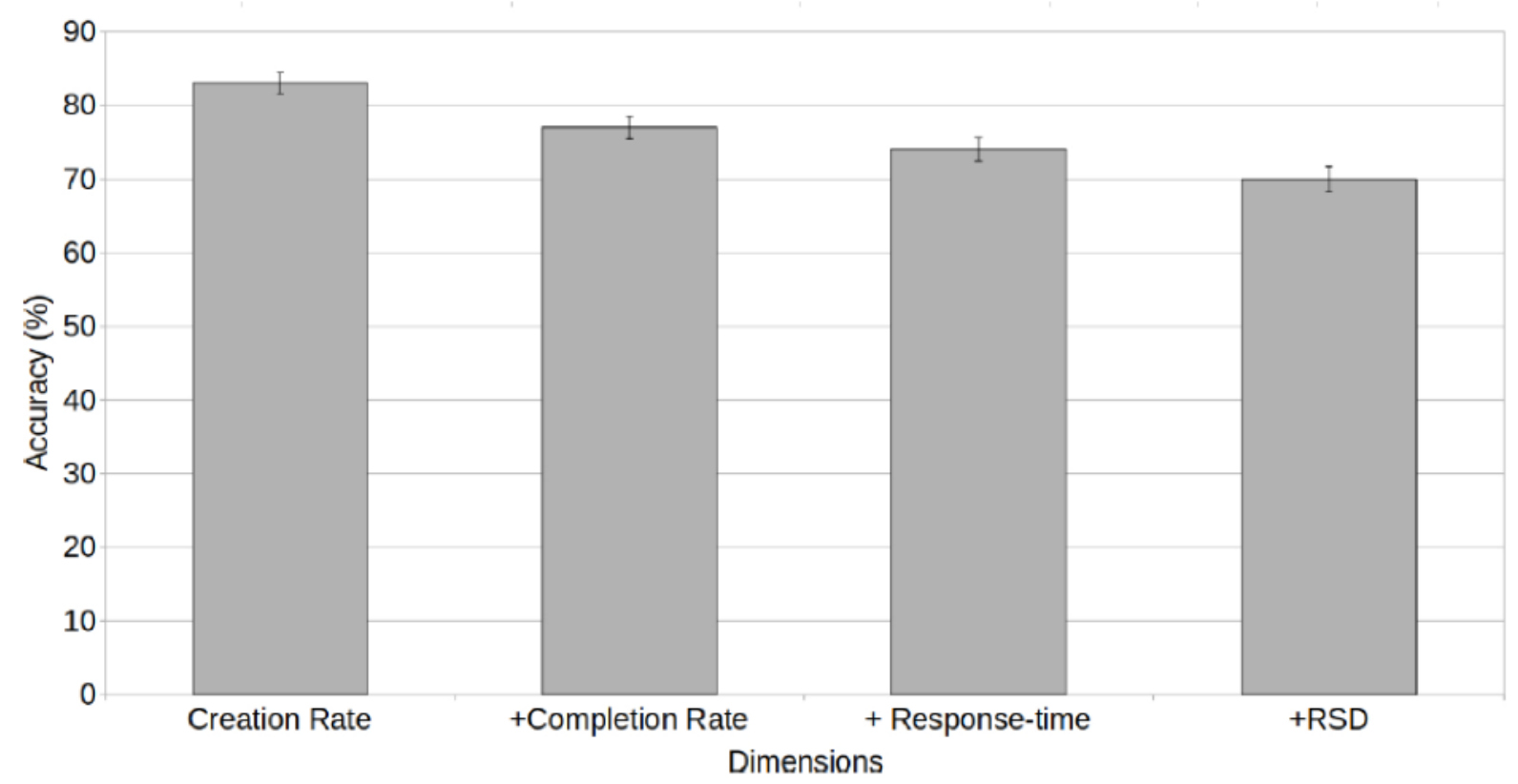

Figure 6.13: Impact of using different container performance statistics on the classification accuracy of the Random Forrest algorithm. From [2]

In short, by constructing a prediction model, container performance metrics can classify Hadoop and Spark workloads effectively.

\subsubsection{Detection of the Workload Transition}

In order to validate whether the container performance metrics can detect the workload transitions effectively, Welch's t-test was leveraged to detect workload transitions in our collected data. In Statistics, Welch's t-test [45] is used to evaluate 
whether the means of two samples are significantly different. The confidence level for rejecting the null hypothesis is $95 \%$ which is a frequent number in statistical tests. The formula of calculation is as follows:

$$
t=\frac{\overline{X_{1}}-\overline{X_{2}}}{\sqrt{\frac{s_{1}^{2}}{N_{1}}+\frac{s_{2}{ }^{2}}{N_{2}}}}
$$

Since the data we collected is time-series data, we employed a time domain technique to evaluate whether the container performance metrics can detect workload transitions and how effective each metric is. The analysis was based on replaying the data in every observation window as a real-time stream. Every fixed number of sequential windows was treated as a computing interval as shown in Figure 6.14. Based on the average and variance calculated in each computing interval, Welch's t-test was leveraged to evaluate whether there is a significant difference between the two consecutive computing intervals.

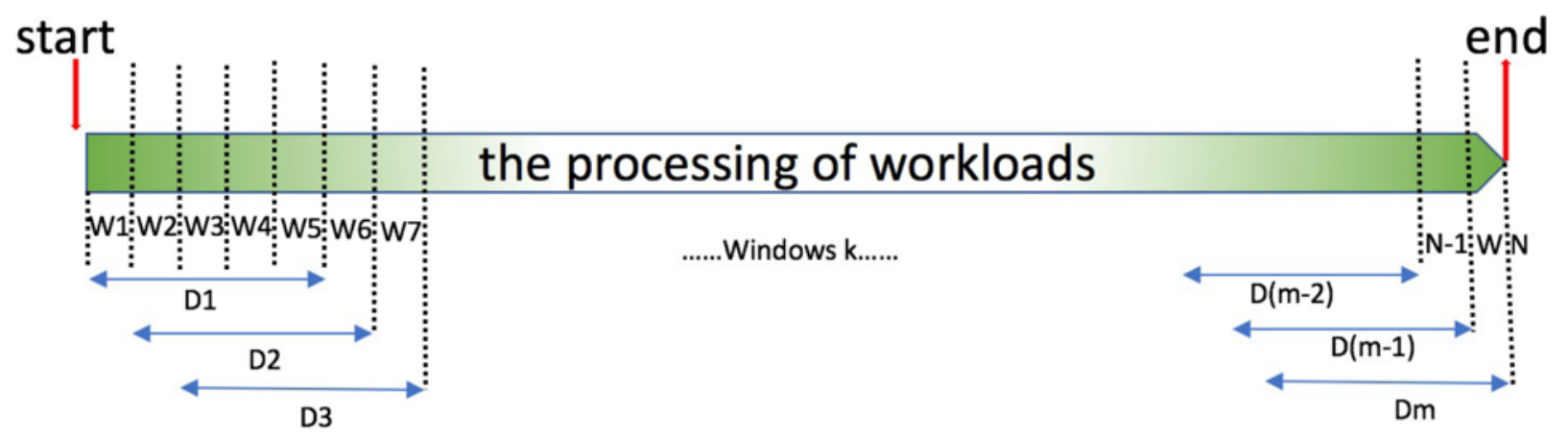

Figure 6.14: Explaination of window

When the statistical results show a significant difference, we considered there was a transition that happened between the two computing intervals. At the same time, according to the logs from the YARN, Hadoop and Spark, we identified the true transition manually. We defined the accuracy of detecting workload transitions as 100 times the number of transitions detected by Welch's t-test divided by the number of transitions identified by the logs.

Based on the data collected from all job flows in our experiments, the detection accuracy was evaluated for each container performance metric separately. 
Figure 6.15 shown the result, RSD has the highest accuracy at $74 \%$.

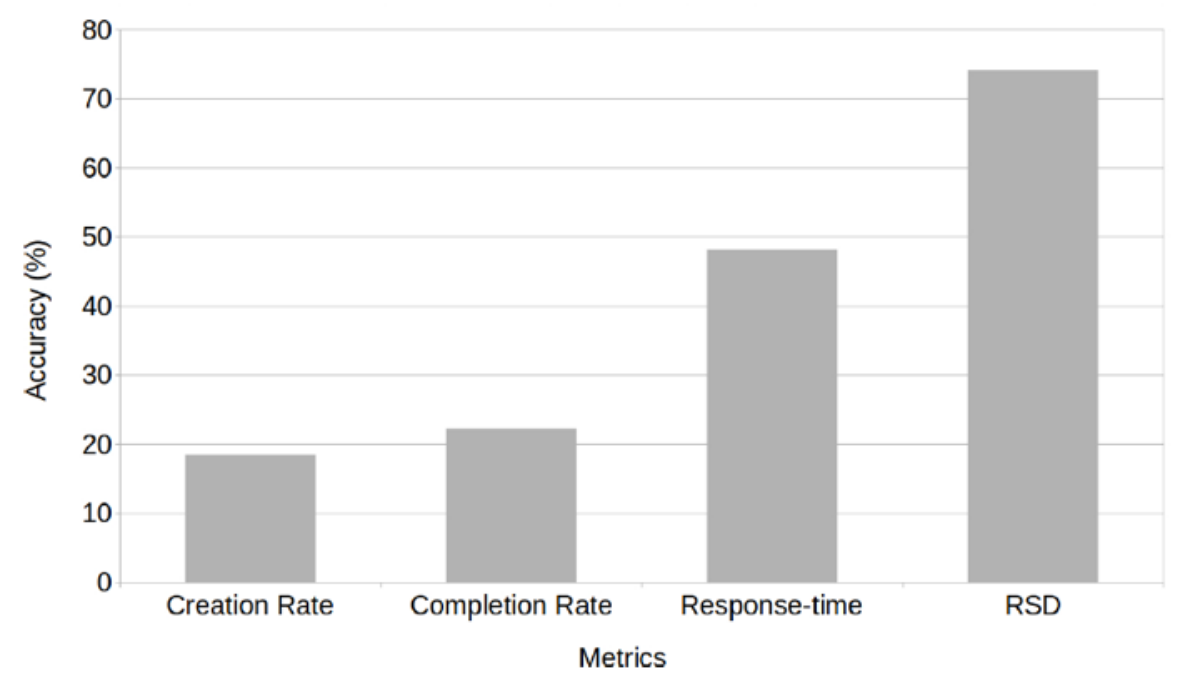

Figure 6.15: Workload transition detection accuracy for different container performance metrics. From [2]

In short, RSD is the most sensitive metric to detect the workload transitions with acceptable accuracy.

\subsection{Ranking of the Metrics}

Based on the conclusions from section 6.1 to 6.3, the four kinds of container performance metrics were ranked. The first one is container creation rate. With this metric only, the prediction model can provide the highest average accuracy to classify Hadoop and Spark workload. The second one is RSD. Even though this metric is not as helpful as container creation rate to identify the workload type, it is the most sensitive metric to detect the workload transitions with acceptable accuracy. The third one is container average response-time. This metric is not as effective as the previous two but can still provide the second highest accuracy in detecting workload transitions. The last one is container completion rate. The metric is not very effective in identifying the workload type and detecting workload transitions. However, it could be used with other metrics to provide valuable suggestions for a tuning strategy. 


\section{Section 7}

\section{Implications for Online Auto-Tuning Systems}

Classifying the workload type and detecting workload transitions are significant to improve the performance of the auto-tuning system. Since Hadoop and Spark request containers in different situations, the tuning strategies should be different. If the automatic tuning system can identify the workload type, it can apply a specific tuning strategy for the current workload. In order to improve efficiency, the system also needs to avoid tuning overhead. Detecting workload transitions is helpful in this situation. When the workload is changed, the requirements of resource allocation will be different which means the tuning should be evoked to search the optimal parameters for the current workload.

We proposed a possible automatic tuning system architecture which is shown in Figure 7.1. This architecture includes three parts: Transition Monitor, Classifier and Tuner. Transition Monitor can collect container performance metrics in real-time and detect the workloads transitions based on the Welch's t-test which we mentioned in section 6.3.2. After the transition has happened, the Classifier component will apply a machine learning algorithm to identify the current workload type. According to the workload type, the Tuner will apply an appropriate tuning strategy, and optimal parameters will be selected.

Based on the analysis of container performance metric values, this design focused on container resource usage, providing a more fine-grained tuning system which is different from a job-by-job tuning system. This system can use container 


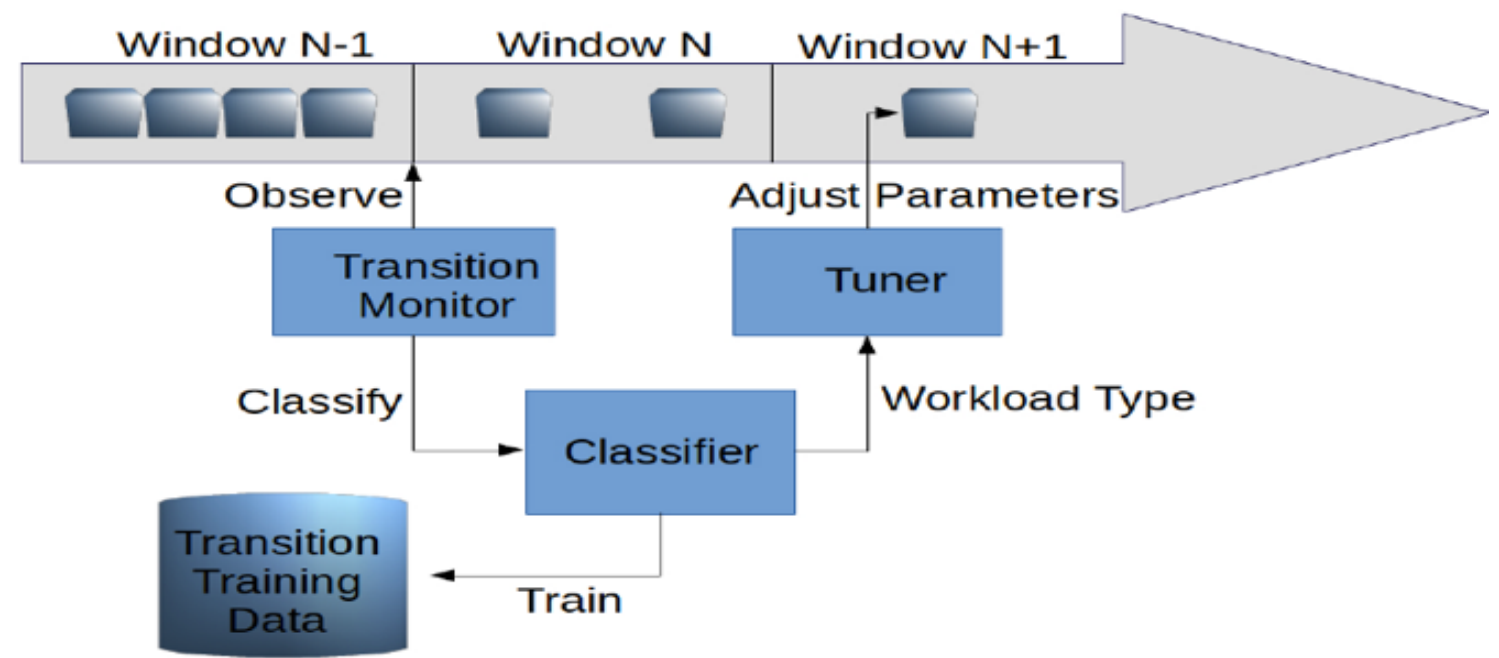

Figure 7.1: A possible automatic tuning system architecture. From [2]

performance metrics to optimize the overall performance of the current running workload regardless of whether it is a single job, single-user job flows, or multi-user job flows.

The main idea for this automatic tuning system is as follows:

1. Choose an appropriate tuning timing by monitoring workload transitions

2. According to the current workload type, select a specific tuning strategy.

Even though our experiments are running on Hadoop and Spark, the main idea we proposed can be adapted for other analytics frameworks as well. 


\section{Section 8}

\section{Conclusions and Future Works}

Conclusions and future work are summarised in this section.

\subsection{Conclusions}

Through the analysis of container performance metrics, this thesis provides further research on improving automatic tuning of Hadoop and Spark by identifying and classifying the workload type, as well as detecting workload transitions. Regardless of what kind of RM is integrated with the analytics framework, the containers will be responsible for executing tasks. Since our research focuses on container performance

metrics, the results of can support working with different kinds of frameworks and the resource managers. Also, this is the first research to detect workload transitions from container perspective.

By presenting the process of analysing twenty-six groups experimental data of Hadoop and Spark workloads, this research proves that container performance metrics could be beneficial to identify the workload type and detect workload transitions. Since the data are collected from single jobs, single user job flows and multi-user job flows, our experiments are more comprehensive, which makes our results more adaptable to real-life scenarios.

According to the data analysis results, a rank of container performance metrics is claimed by this thesis. Based on our machine learning workload classifier, container creation rate is the most useful metric to distinguish Hadoop workload from Spark with an average prediction accuracy of $84 \%$. RSD is the most sensitive 
metric to detect the transitions with an average accuracy of $74 \%$.

Our results could be used to improve the efficiency of current automatic online tuning systems. The possible prototype proposed in this research can choose appropriate tuning time by monitoring workload transitions and selecting a specific tuning strategy for the current workload.

\subsection{Future Work}

Possible future work we can consider are as follows:

1. Improve Kermit by integrating with the results of this research work.

2. Try to improve the accuracy of workload classification. We can try to include CPU, memory, disk and network usage into input data when we train the machine learning classifier.

3. Try the fine-grain workload type classification. For example, distinguish Spark SQL queries from Spark RDD operations.

4. Do more experiments and analyse the characteristic of workloads. According to different features of workloads, we could try to design specific tuning strategies.

5. Try to improve the accuracy of detecting workloads transitions 


\section{List of References}

[1] M. Genkin, F. Dehne, M. Pospelova, Y. Chen, and P. Navarro, "Automatic, on-line tuning of yarn container memory and cpu parameters," in High Performance Computing and Communications; IEEE 14th International Conference on Smart City; IEEE 2nd International Conference on Data Science and Systems (HPCC/SmartCity/DSS), 2016 IEEE 18th International Conference on, pp. 317-324, IEEE, 2016.

[2] M. Genkin, F. Dehne, P. Navarro, and S. Zhou, "Machine-learning based spark and hadoop workload classification using container performance patterns," in International Symposium on Benchmarking, Measuring and Optimizing proceedings in progress, 2018.

[3] S. Huang, J. Huang, Y. Liu, L. Yi, and J. Dai, "Hibench: A representative and comprehensive hadoop benchmark suite," in Proc. ICDE Workshops, 2010.

[4] Wikipedia, "Data science: An introduction/a history of data science." https://en.wikibooks.org/wiki/Data_Science:_An_Introduction/ A_History_of_Data_Science\#cite_note-1. Accessed: 2019-02-01.

[5] V. P. Navarro Belmonte, Improving Real Time Tuning on YARN. PhD thesis, Carleton University, 2018.

[6] M. Pospelova, Real Time Autotuning for MapReduce on Hadoop/YARN. PhD thesis, Carleton University, 2015.

[7] X. Ding, Y. Liu, and D. Qian, "Jellyfish: Online performance tuning with adaptive configuration and elastic container in hadoop yarn," in Parallel and Distributed Systems (ICPADS), 2015 IEEE 21st International Conference on, pp. 831-836, IEEE, 2015.

[8] M. Li, L. Zeng, S. Meng, J. Tan, L. Zhang, A. R. Butt, and N. Fuller, "Mronline: Mapreduce online performance tuning," in Proceedings of the 23rd international symposium on High-performance parallel and distributed computing, pp. 165-176, ACM, 2014.

[9] S. Ghemawat, H. Gobioff, and S.-T. Leung, The Google file system, vol. 37. ACM, 2003.

[10] J. Dean and S. Ghemawat, "Mapreduce: simplified data processing on large clusters," Communications of the ACM, vol. 51, no. 1, pp. 107-113, 2008. 
[11] Hortonworks, "Apache hadoop yarn concepts and applications." https://hortonworks.com/blog/ apache-hadoop-yarn-concepts-and-applications/. Accessed: 2019-0201.

[12] Wikipedia, "Apache spark." https://en.wikipedia.org/wiki/Apache_Spark. Accessed: 2019-02-01.

[13] Spark, "Cluster mode overview." https://spark.apache.org/docs/latest/ cluster-overview.html. Accessed: 2019-02-01.

[14] Cloudera, "Spark application overview." https://www.cloudera.com/ documentation/enterprise/5-6-x/topics/cdh_ig_spark_apps.html. Accessed: 2019-02-01.

[15] D. Flair, "Apache spark executor for executing spark tasks." https:// data-flair.training/blogs/spark-executor/. Accessed: 2019-02-01.

[16] H. Karau, A. Konwinski, P. Wendell, and M. Zaharia, Learning spark: lightningfast big data analysis. " O'Reilly Media, Inc.", 2015.

[17] Q. Chen, M. Guo, Q. Deng, L. Zheng, S. Guo, and Y. Shen, "Hat: historybased auto-tuning mapreduce in heterogeneous environments," The Journal of Supercomputing, vol. 64, no. 3, pp. 1038-1054, 2013.

[18] G. Liao, K. Datta, and T. L. Willke, "Gunther: Search-based auto-tuning of mapreduce," in European Conference on Parallel Processing, pp. 406-419, Springer, 2013.

[19] H. Herodotou, H. Lim, G. Luo, N. Borisov, L. Dong, F. B. Cetin, and S. Babu, "Starfish: a self-tuning system for big data analytics.," in Cidr, vol. 11, pp. 261$272,2011$.

[20] C. A. Schaefer, V. Pankratius, and W. F. Tichy, "Atune-il: An instrumentation language for auto-tuning parallel applications," in European Conference on Parallel Processing, pp. 9-20, Springer, 2009.

[21] M. J. Cafarella and C. Ré, "Manimal: relational optimization for data-intensive programs," in Procceedings of the 13th International Workshop on the Web and Databases, p. 10, ACM, 2010.

[22] J. Liu, N. Ravi, S. Chakradhar, and M. Kandemir, "Panacea: Towards holistic optimization of mapreduce applications," in Proceedings of the Tenth International Symposium on Code Generation and Optimization, pp. 33-43, ACM, 2012.

[23] N. Yigitbasi, T. L. Willke, G. Liao, and D. Epema, "Towards machine learningbased auto-tuning of mapreduce," in 2013 IEEE 21st International Symposium on Modelling, Analysis and Simulation of Computer and Telecommunication Systems, pp. 11-20, IEEE, 2013.

[24] E. C. de Almeida, Y. Le Traon, et al., "Intra-query adaptivity for mapreduce query processing systems," in Proceedings of the 18th International Database Engineering \& Applications Symposium, pp. 380-381, ACM, 2014. 
[25] R. Zhang, M. Li, and D. Hildebrand, "Finding the big data sweet spot: Towards automatically recommending configurations for hadoop clusters on docker containers," in 2015 IEEE International Conference on Cloud Engineering (IC2E), pp. 365-368, IEEE, 2015.

[26] K. Wang, B. Tan, J. Shi, and B. Yang, "Automatic task slots assignment in hadoop mapreduce," in Proceedings of the 1st Workshop on Architectures and Systems for Big Data, pp. 24-29, ACM, 2011.

[27] G. Wang, J. Xu, and B. He, "A novel method for tuning configuration parameters of spark based on machine learning," in High Performance Computing and Communications; IEEE 14th International Conference on Smart City; IEEE 2nd International Conference on Data Science and Systems (HPCC/SmartCity/DSS), 2016 IEEE 18th International Conference on, pp. 586-593, IEEE, 2016.

[28] Z. Jia, C. Xue, G. Chen, J. Zhan, L. Zhang, Y. Lin, and P. Hofstee, "Auto-tuning spark big data workloads on power8: Prediction-based dynamic smt threading," in Parallel Architecture and Compilation Techniques (PACT), 2016 International Conference on, pp. 387-400, IEEE, 2016.

[29] Y. Zhao, F. Hu, and H. Chen, "An adaptive tuning strategy on spark based on inmemory computation characteristics," in Advanced Communication Technology (ICACT), 2016 18th International Conference on, pp. 484-488, IEEE, 2016.

[30] M. Wasi-Ur-Rahman, N. S. Islam, X. Lu, D. Shankar, and D. K. Panda, "Mradvisor: A comprehensive tuning tool for advising hpc users to accelerate mapreduce applications on supercomputers," in Computer Architecture and High Performance Computing (SBAC-PAD), 2016 28th International Symposium on, pp. 198-205, IEEE, 2016.

[31] Z. Jia, J. Zhan, L. Wang, R. Han, S. A. McKee, Q. Yang, C. Luo, and J. Li, "Characterizing and subsetting big data workloads," in Workload Characterization (IISWC), 2014 IEEE International Symposium on, pp. 191-201, IEEE, 2014.

[32] W. D. Mulia, N. Sehgal, S. Sohoni, J. M. Acken, C. L. Stanberry, and D. J. Fritz, "Cloud workload characterization," IETE Technical Review, vol. 30, no. 5, pp. 382-397, 2013.

[33] A. J. Awan, M. Brorsson, V. Vlassov, and E. Ayguade, "Micro-architectural characterization of apache spark on batch and stream processing workloads," in Big Data and Cloud Computing (BDCloud), Social Computing and Networking (SocialCom), Sustainable Computing and Communications (SustainCom)(BDCloud-SocialCom-SustainCom), 2016 IEEE International Conferences on, pp. 59-66, IEEE, 2016.

[34] A. K. Mishra, J. L. Hellerstein, W. Cirne, and C. R. Das, "Towards characterizing cloud backend workloads: insights from google compute clusters," $A C M$ SIGMETRICS Performance Evaluation Review, vol. 37, no. 4, pp. 34-41, 2010. 
[35] I. S. Moreno, P. Garraghan, P. Townend, and J. Xu, "An approach for characterizing workloads in google cloud to derive realistic resource utilization models," in Service Oriented System Engineering (SOSE), 2013 IEEE 7th International Symposium on, pp. 49-60, IEEE, 2013.

[36] Apache, "Hadoop." https://hadoop.apache.org. Accessed: 2019-02-01.

[37] Apache, "Spark." https://spark.apache.org. Accessed: 2019-02-01.

[38] Intel, "Hibench." https://github.com/intel-hadoop/HiBench/tree/6.0. Accessed: 2019-02-01.

[39] O. OMalley, "Terabyte sort on apache hadoop," Yahoo, available online at: http://sortbenchmark. org/Yahoo-Hadoop. pdf,(May), pp. 1-3, 2008.

[40] M. G. Noll, "Benchmarking and stress testing an hadoop cluster with terasort, testdfsio." http://www.michael-noll.com/blog/2011/04/09/ benchmarking-and-stress-testing-an-hadoop-cluster-with-terasort-testdfsio-nnb Accessed: 2019-02-01.

[41] Databricks, "Spark-perf." https://github.com/databricks/spark-perf/. Accessed: 2019-02-01.

[42] IBM, "Sparkmultiuserbenchmark." https://github.com/IBM/ SparkMultiuserBenchmark/. Accessed: 2019-02-01.

[43] Wikipedia, "Correlation coefficient." https://en.wikipedia.org/wiki/ Correlation_coefficient. Accessed: 2019-02-01.

[44] E. Solutions, "Accuracy, precision, recall, f1 score: Interpretation of performance measures." https://blog.exsilio.com/all/ accuracy-precision-recall-f1-score-interpretation-of-performance-measures/. Accessed: 2019-02-01.

[45] S. H. To, "Welch's test for unequal variances." https://www. statisticshowto. datasciencecentral.com/welchs-test-for-unequal-variances/. Accessed: 2019-02-01. 